\title{
Fully-Discrete Finite Element Approximation for a Family of Degenerate Parabolic Problems
}

\section{Ramiro Acevedo ${ }^{a}$, Christian Gómez ${ }^{a}$ and Bibiana López-Rodríguez ${ }^{b}$}

\author{
${ }^{a}$ Universidad del Cauca, Departamento de Matemáticas \\ Calle 5 \# 4-70, Popayán (Cauca), Colombia \\ ${ }^{b}$ Universidad Nacional de Colombia sede Medellín, Escuela de Matemáticas \\ Carrera 6 \# 59a-110, Medellín (Antioquia), Colombia \\ E-mail(corresp.): rmacevedo@unicauca.edu.co \\ E-mail: christiancamilo@unicauca.edu.co \\ E-mail: blopezr@unal.edu.co
}

Received May 29, 2020; revised December 16, 2021; accepted December 16, 2021

\begin{abstract}
The aim of this work is to show an abstract framework to analyze the numerical approximation by using a finite element method in space and a BackwardEuler scheme in time of a family of degenerate parabolic problems. We deduce sufficient conditions to ensure that the fully-discrete problem has a unique solution and to prove quasi-optimal error estimates for the approximation. Finally, we show a degenerate parabolic problem which arises from electromagnetic applications and deduce its well-posedness and convergence by using the developed abstract theory, including numerical tests to illustrate the performance of the method and confirm the theoretical results.

Keywords: parabolic degenerate equations, parabolic-elliptic equations, finite element method, backward Euler scheme, fully-discrete approximation, error estimates, eddy current model.
\end{abstract}

AMS Subject Classification: $35 \mathrm{~K} 65 ; 35 \mathrm{~K} 90$.

\section{Introduction}

We will call degenerate parabolic equation an elliptic-parabolic equation of the form ( [20, Chapter III], [22, Section 44], [16]):

$$
\frac{d}{d t}(R u(t))+A(t) u(t)=f(t)
$$

Copyright (c) 2022 The Author(s). Published by Vilnius Gediminas Technical University

This is an Open Access article distributed under the terms of the Creative Commons Attribution License (http://creativecommons.org/licenses/by/4.0/), which permits unrestricted use, distribution, and reproduction in any medium, provided the original author and source are credited. 
where $R$ is a linear, bounded and monotone operator independent of time and $(A(t))_{t \in[0, T]}$ is a family of linear and bounded operators. They arise in several applications, for instance in the study of eddy currents in electromagnetic field theory (see $[5,13,24]$ ).

Results about existence and uniqueness of solutions for some degenerate parabolic equations have been widely studied. In [11] Kuttler and Kenneth show results concerning existence, uniqueness and regularity of equations of the form (1.1), where $R$ is non-invertible and $A$ is a linear operator independent of the time. Sufficient conditions to ensure the existence and uniqueness of solutions of (1.1), even when $R$ depends on the time, are shown by Showalter [20] (see also [19]). Moreover, the existence and uniqueness of the solutions for the case of the family of operators $A$ can be non-linear, has been analyzed in $[10,12,14]$.

Among the numerical methods found in the literature to compute the approximated solution of classical parabolic partial differential equation, the finite element method (with some time-stepping scheme) is one of the more extended. We can cite the book by V. Thomée [21] as a classical reference about this topic. Moreover, books dedicate to the finite element approximation for partial differential equations, devote at least one chapter to the analysis of the numerical approximation of parabolic equations (see, for instance, [9] and [17]). In fact, the developed theory for the approximation of parabolic equations by the finite element method, is mainly presented for a general heat-like equation, i.e., to approximate the solution of a general parabolic problem of the form:

$$
\frac{d u}{d t}+\mathcal{L} u=f
$$

with $\mathcal{L}$ is a coercive differential operator of the second order.

The mathematical analysis for the numerical approximations by finite element methods, including existence and uniqueness of the discrete solutions and quasi-optimal error estimates, has been only performed for particular degenerate parabolic equations. For instance, Zlamal [24] has studied the approximation of solution for a two-dimensional eddy current problem in a bounded domain, MacCamy and Suri [13] have proposed a FEM-BEM coupling for the formulation analyzed in [24], and a formulation for an axisymmetric eddy current problem was studied by Bermudez et al [5]. The formulations studied in all these references can be expressed as particular cases of problem (1.1). Nevertheless, to the best knowledge of the authors, there is not an abstract general theory that allows to deduce the mathematical analysis of these approximations as particular applications of that theory.

The main goal of this article is precisely to provide a general theory for the mathematical analysis of a fully-discrete finite element approximation for an abstract degenerate parabolic equation. To this end, we consider a fully discrete approximation for a Cauchy problem associated to equation (1.1), by using a finite element method in space and a Backward-Euler scheme in time. We show sufficient conditions for the spaces and the family of operators, to guarantee existence and uniqueness of the fully-discrete solutions by assuming that the time step is sufficiently small. Furthermore, we prove quasi-optimal 
error estimates for this fully discretized scheme by adapting the approximation theory for classical parabolic equations to the abstract degenerate case. Moreover, since a good discrete approximation for the time-derivative of the solution is relevant for the applications, we prove that this time derivative can be approximated with quasi-optimal error estimates.

The outline of the paper is as follows: Section 2 is devoted to show some concepts about spaces for evolutive problems and the abstract framework for degenerate parabolic equations and their well-posedness are recalled in Section 3. The corresponding analysis for the fully-discrete approximation of problem by using finite element method in space and a backward Euler scheme in time, is presented in Section 4 and the results ensuring the quasi-optimal convergence of the approximation method are shown in Section 5. Furthermore, the application of the theory to an eddy current model is studied in Section 6, where we deduce its well-posedness and theoretical convergence by using the developed abstract theory. Finally, we show some numerical results that confirm the expected convergence of the method according to the theory.

\section{Hilbert functional spaces for evolutive problems}

Let us first review some basic concepts about functional analysis which are useful in dealing with time-dependent functions. A complete and detailed presentation of the concepts that we indicate in this section can be founded, for instance, in [23, Sections 23.2-23.6]. More precisely, we need to introduce spaces of functions defined on a bounded time interval $(0, T)$ (where $T>0$ is a fixed time) and with values in separable Hilbert space $X$. We will denote by $\|\cdot\|_{X},(\cdot, \cdot)_{X}$ and $\langle\cdot, \cdot\rangle_{X}$, the norm, the inner product and duality pairing in $X$. We use the notation $\mathcal{C}^{0}([0, T] ; X)$ for the space consisting of all continuous functions $f:[0, T] \rightarrow X$. More generally, for any $k \in \mathbb{N}, \mathcal{C}^{k}([0, T] ; X)$ denotes the subspace of $\mathcal{C}^{0}([0, T] ; X)$ of all functions $f$ with (strong) derivatives of order at most $k$ in $\mathcal{C}^{0}([0, T] ; X)$, i.e.,

$$
\mathcal{C}^{k}([0, T] ; X):=\left\{f \in \mathcal{C}^{0}([0, T] ; X): \quad \frac{d^{j} f}{d t^{j}} \in \mathcal{C}^{0}([0, T] ; X), \quad 1 \leq j \leq k\right\} .
$$

A classical result of functional analysis states $\mathcal{C}^{k}([0, T] ; X)$ is a Banach space with the norm

$$
\|f\|_{\mathcal{C}^{k}([0, T] ; X)}:=\sup _{t \in[0, T]} \sum_{j=0}^{k}\left\|\frac{d^{j} f}{d t^{j}}(t)\right\|_{X} .
$$

We also consider the space $\mathrm{L}^{2}(0, T ; X)$ of classes of functions $f:(0, T) \rightarrow X$ that are Böchner-measurable whose norm in $X$ belongs to $\mathrm{L}^{2}(0, T)$, i.e.,

$$
\|f\|_{\mathrm{L}^{2}(0, T ; X)}^{2}:=\int_{0}^{T}\|f(t)\|_{X}^{2} d t<+\infty .
$$

The space $\mathrm{L}^{2}(0, T ; X)$ is a Hilbert space with the norm $\|\cdot\|_{\mathrm{L}^{2}(0, T ; X)}$. Furthermore, the dual space of $\mathrm{L}^{2}(0, T ; X)$ can be identified with the space $\mathrm{L}^{2}\left(0, T ; X^{\prime}\right)$ as shown in the following result. 
Proposition 1 [Dual space of $\mathrm{L}^{2}(0, T ; X)$ ]. Let $X$ be a separable Hilbert space. For any $f \in \mathrm{L}^{2}(0, T ; X)^{\prime}$ there exists a unique $v_{f} \in \mathrm{L}^{2}\left(0, T ; X^{\prime}\right)$ satisfying

$$
\langle f, w\rangle=\int_{0}^{T}\left\langle v_{f}(t), w(t)\right\rangle_{X} d t, \quad \forall w \in \mathrm{L}^{2}(0, T ; X) .
$$

Moreover, the map $f \mapsto v_{f}$ is a linear bijection which preserves the norm, i.e.,

$$
\|f\|_{\left(L^{2}(0, T ; X)\right)^{\prime}}=\left\|v_{f}\right\|_{L^{2}\left(0, T ; X^{\prime}\right)}, \quad \forall f \in\left(L^{2}(0, T ; X)\right)^{\prime} .
$$

Proof. See, for instance, [23, Proposition 23.7].

The analysis of evolutive differential problems require functional spaces involving time-derivatives. Let $X$ and $Y$ be two separable Hilbert spaces such that $X \subset Y$ with continuous and dense embedding. Let $X^{\prime}$ the dual space of $X$ with respect to the pivot space $Y$. More precisely, $Y$ will be identified with its dual $Y^{\prime}$ by the Riesz map and we have $X \hookrightarrow Y \hookrightarrow X^{\prime}$ with the identity

$$
\langle w, v\rangle_{X}=(w, v)_{Y} \quad \forall w \in Y \subset X^{\prime} \quad \forall v \in X .
$$

We will denote by $\mathrm{W}^{1,2}\left(0, T ; X, X^{\prime}\right)$ the functional space given by

$$
\mathrm{W}^{1,2}\left(0, T ; X, X^{\prime}\right):=\left\{v \in \mathrm{L}^{2}(0, T ; X): \frac{d v}{d t} \in \mathrm{L}^{2}\left(0, T ; X^{\prime}\right)\right\},
$$

where $\frac{d v}{d t}$ is the generalized time-derivative of $v$ characterized by

$$
\int_{0}^{T}\left\langle\frac{d v}{d t}(t), w\right\rangle_{X} \varphi(t) d t=-\int_{0}^{T}(v(t), w)_{Y} \varphi^{\prime}(t) d t \quad \forall w \in X \quad \forall \varphi \in C_{0}^{\infty}(0, T) .
$$

The symbols $\frac{d v}{d t}, \partial_{t} v$ and $v^{\prime}$ will be used indistinctly along the text to denote the generalized time-derivative of $v$. It is well known that $\mathrm{W}^{1,2}\left(0, T ; X, X^{\prime}\right)$ endowed with the norm

$$
\|v\|_{\mathrm{W}^{1,2}\left(0, T ; X, X^{\prime}\right)}:=\|v\|_{L^{2}(0, T ; X)}+\left\|\frac{d v}{d t}\right\|_{L^{2}\left(0, T ; X^{\prime}\right)}
$$

is a Banach space and $\mathrm{W}^{1,2}\left(0, T ; X, X^{\prime}\right) \subset \mathcal{C}^{0}([0, T] ; Y)$ with a continuous embedding (see, for instance, [23, Proposition 23.23]).

Let $k \in \mathbb{N}$. The generalized time-derivative of order $k$ of $v \in \mathrm{L}^{2}(0, T ; X)$, denoted by $\frac{d^{k} v}{d t^{k}}$, can be defined inductively. Hence, we can consider the space

$$
\mathrm{H}^{k}(0, T ; X):=\left\{v \in \mathrm{L}^{2}(0, T ; X): \frac{d^{j} v}{d t^{j}} \in \mathrm{L}^{2}(0, T ; X), j=1, \ldots, k\right\},
$$

which is a Banach space with the norm

$$
\|v\|_{\mathrm{H}^{k}(0, T ; X)}:=\sum_{j=0}^{k}\left\|\frac{d^{j} v}{d t^{j}}\right\|_{L^{2}(0, T ; X)} .
$$

Furthermore, the embedding $\mathrm{H}^{k}(0, T ; X) \subset \mathcal{C}^{k-1}([0, T] ; X)$ is continuous for any $k \in \mathbb{N}$. 


\section{The degenerate parabolic problem}

Let $X$ and $Y$ be two real separable Hilbert spaces such that $X \subset Y$ with continuous and dense embedding. We denote by $(\cdot, \cdot)_{X}$ and $(\cdot, \cdot)_{Y}$ the inner products on $X$ and $Y$ respectively and $\|\cdot\|_{X},\|\cdot\|_{Y}$ the corresponding norms. Furthermore, $\langle\cdot, \cdot\rangle_{X}$ and $\langle\cdot, \cdot\rangle_{Y}$ denote respectively the duality paring of $X$ and $Y$ and their corresponding dual spaces. Let $R: Y \rightarrow Y^{\prime}$ a linear and bounded operator. Let $T>0$, for any $t \in[0, T]$, let us consider a linear and bounded operator $A(t): X \rightarrow X^{\prime}$ such that

$$
\begin{aligned}
& t \in[0, T] \mapsto\langle A(t) v, w\rangle_{X} \in \mathbb{R} \text { is measurable, } \forall v, w \in X, \\
& \exists M>0 \text { constant s.t }\|A(t)\|_{\mathrm{L}\left(X, X^{\prime}\right)} \leq M, \quad \forall t \in[0, T] .
\end{aligned}
$$

For any function $w:[0, T] \rightarrow X$ we will use the following notation

$$
R w(t):=R(w(t)), \quad A(t) w(t):=A(t)(w(t)) .
$$

Then, given $f \in \mathrm{L}^{2}\left(0, T ; X^{\prime}\right)$ and $u_{0} \in Y$, the degenerate parabolic problem can read as follows.

Problem 1. Find $u \in \mathrm{L}^{2}(0, T ; X)$ such that:

$$
\begin{array}{rlrl}
\frac{d}{d t}\langle R u(t), v\rangle_{Y}+\langle A(t) u(t), v\rangle_{X} & =\langle f(t), v\rangle_{X}, & \forall v & \in X, \\
\langle R u(0), v\rangle_{Y} & =\left\langle R u_{0}, v\right\rangle_{Y}, \quad \forall v \in Y .
\end{array}
$$

The first identity in Problem 1 is given in the space of the distributions $\mathcal{D}^{\prime}(0, T)$, i.e., this equation is equivalent to

$$
-\int_{0}^{T}\langle R u(t), v\rangle_{Y} \varphi^{\prime}(t) d t+\int_{0}^{T}\langle A(t) u(t), v\rangle_{X} \varphi(t) d t=\int_{0}^{T}\langle f(t), v\rangle_{X} \varphi(t) d t
$$

for all $v \in X$ and $\varphi \in C_{0}^{\infty}(0, T)$. Moreover, Problem 1 can be formulated as any of the following two equivalent problems.

Problem 2. Find $u \in \mathrm{L}^{2}(0, T ; X)$ such that

$$
-\int_{0}^{T}\left\langle R u(t), v^{\prime}(t)\right\rangle_{Y} d t+\int_{0}^{T}\langle A(t) u(t), v(t)\rangle_{X} d t=\int_{0}^{T}\langle f(t), v(t)\rangle_{X} d t+\left\langle R u_{0}, v(0)\right\rangle_{Y},
$$

for all $v \in \mathrm{L}^{2}(0, T ; X) \cap H^{1}(0, T ; Y)$ with $v(T)=0$.

Problem 3. Find $u \in \mathrm{L}^{2}(0, T ; X)$ satisfying

$$
\begin{aligned}
\frac{d}{d t} R u(\cdot)+A(\cdot) u(\cdot) & =f(\cdot) \quad \text { in } \quad \mathrm{L}^{2}\left(0, T ; X^{\prime}\right), \\
R u(0) & =R u_{0} \quad \text { in } \quad Y^{\prime} .
\end{aligned}
$$

Remark 1. The first equation in Problem 3 implies that $R u(\cdot) \in H^{1}\left(0, T ; X^{\prime}\right)$, consequently the function $t \mapsto R u(t)$ is absolutely continuous in $X^{\prime}$ and in particular $R u(0) \in X^{\prime}$. Moreover, since the inclusion $X \subset Y$ is dense and continuous, the inclusion $Y^{\prime} \subset X^{\prime}$ is also dense and continuous and therefore, by recalling that $R u_{0} \in Y^{\prime}$, the initial condition given by the second equation of Problem 3 has meaning, which is equivalent to the second equation of Problem 1. 
Remark 2. Problem 1 allows some arbitrariness in the initial data $u_{0}$. More precisely, if $u_{0}$ is replaced by $u_{0}+z$, where $z$ is an arbitrary element of ker $R$, the initial condition given by the second equation in Problem 1 does not change and therefore neither does the solution of this problem.

In order to obtain the well-posedness result for Problem 1 (and equivalently for Problem 2 and Problem 3), we need to recall the following definition; see [20, Section III.3].

Definition 1 . Let $Z$ be a real separable Hilbert space and $\mathcal{G}:=\{G(t): Z \rightarrow$ $\left.Z^{\prime}: t \in[0, T]\right\}$ be a family of linear and bounded operators. $\mathcal{G}$ is called monotone if $\langle G(t) v, v\rangle_{Z} \geq 0$ for any $v \in Z$ and for any $t \in[0, T]$. $\mathcal{G}$ is called self-adjoint, if $\langle G(t) u, v\rangle_{Z}=\langle G(t) v, u\rangle_{Z}$ for any $u, v \in Z$ and for any $t \in[0, T]$. Similarly, $\mathcal{G}$ is called regular if for each $u, v \in Z$ the map $t \mapsto\langle G(t) u, v\rangle_{Z}$ is absolutely continuous on $[0, T]$ and there exists a function $k:(0, T) \rightarrow \mathbb{R}$ belongs to $L^{1}(0, T)$, which satisfies

$$
\left|\frac{d}{d t}\langle G(t) u, v\rangle_{Z}\right| \leq k(t)\|u\|_{Z}\|v\|_{Z}, \quad \forall u, v \in Z \quad \text { a.e. } t \in[0, T] .
$$

The following result shows sufficient conditions to obtain the existence and uniqueness of solution for Problem 1 and its proof can be founded in [20, Proposition III.3.2 and III.3.3].

Theorem 1. Assume that the operator $R$ is monotone, self-adjoint, and there exist constants $\lambda>0$ and $\alpha>0$ such that

$$
\lambda\langle R v, v\rangle_{Y}+\langle A(t) v, v\rangle_{X} \geq \alpha\|v\|_{X}^{2}, \quad \forall v \in X, \quad \forall t \in[0, T] .
$$

Then, there exists a solution of Problem 1 and it satisfies

$$
\|u\|_{\mathrm{L}^{2}(0, T ; X)} \leq C\left(\|f\|_{\mathrm{L}^{2}\left(0, T ; X^{\prime}\right)}^{2}+\left\langle R u_{0}, u_{0}\right\rangle_{Y}\right)^{\frac{1}{2}}
$$

for some constant $C>0$. Furthermore, if $A(t)$ is a regular family of self-adjoint operators, then the solution of Problem 1 is unique.

\section{Fully-discrete approximation for degenerate parabolic problem}

In this section we present the fully-discrete approximation for the degenerate parabolic problem which was introduced in the previous section. For this purpose, we assume that the family of operators $A(t)$ and the operator $R$ satisfy the sufficient conditions given in Theorem 1 to guarantee the existence and uniqueness of solution of Problem 1.

The fully discrete approximation will be obtained by using the finite element method in space and a backward-Euler scheme in time. Let $\left\{X_{h}\right\}_{h>0}$ be a sequence of finite-dimensional subspaces of $X$ and let $t_{n}:=n \Delta t, n=0, \ldots, N$, be a uniform partition of $[0, T]$ with a time-step $\Delta t:=T / N$. 
For any finite sequence $\left\{\theta^{n}: n=0, \ldots, N\right\}$ we denote $\bar{\partial} \theta^{n}:=\frac{\theta^{n}-\theta^{n-1}}{\Delta t}$, $n=1, \ldots, N$.

Let $u_{0, h} \in X_{h}$ and assume that $f \in \mathcal{C}^{0}\left([0, T] ; X^{\prime}\right)$, then the fully-discrete approximation of Problem 1 reads as follows.

Problem 4. Find $u_{h}^{n} \in X_{h}, n=1, \ldots, N$, such that

$$
\begin{aligned}
\left\langle R \bar{\partial} u_{h}^{n}, v\right\rangle_{Y}+\left\langle A\left(t_{n}\right) u_{h}^{n}, v\right\rangle_{X} & =\left\langle f\left(t_{n}\right), v\right\rangle_{X}, \quad \forall v \in X_{h}, \\
u_{h}^{0} & =u_{0, h} .
\end{aligned}
$$

We can easily check that in each step $n=1, \ldots, N, u_{h}^{n}$ is computed as the solution of the following problem: find $u_{h}^{n} \in X_{h}$ such that

$$
\mathcal{A}_{n}\left(u_{h}^{n}, v\right)=F_{n}(v), \quad \forall v \in X_{h},
$$

where $\mathcal{A}_{n}$ and $F_{n}$ are defined by

$$
\begin{gathered}
\mathcal{A}_{n}(v, w):=\langle R v, w\rangle_{Y}+\Delta t\left\langle A\left(t_{n}\right) v, w\right\rangle_{X}, \quad \forall v, w \in X_{h}, \\
F_{n}(v):=\Delta t\left\langle f\left(t_{n}\right), v\right\rangle_{X}+\left\langle R u_{h}^{n-1}, v\right\rangle_{Y}, \quad \forall v \in X_{h} .
\end{gathered}
$$

We will use the Lax-Milgram Lemma to deduce the existence and uniqueness of solution of Problem 4 for each $n=1, \ldots, N$. Since $F_{n}$ is linear and bounded and $\mathcal{A}_{n}$ is bilinear and bounded, we need to prove that $\mathcal{A}_{n}$ is elliptic in $X_{h}$. In fact, if we assume that $0<\Delta t \leq 1 / \lambda$, for any $v \in X_{h}$ we have

$$
\mathcal{A}_{n}(v, v)=\langle R v, v\rangle_{Y}+\Delta t\left\langle A\left(t_{n}\right) v, v\right\rangle_{X} \geq \Delta t\left[\lambda\langle R v, v\rangle_{Y}+\left\langle A\left(t_{n}\right) v, v\right\rangle_{X}\right],
$$

then, from (3.1) it follows that

$$
\mathcal{A}_{n}(v, v) \geq \alpha \Delta t\|v\|_{X}^{2}, \quad \forall v \in X_{h}
$$

Consequently, we have the following result about the existence and uniqueness of solution for the fully-discrete Problem 4.

Theorem 2. Assume that the family of operators $A(t)$ and the operator $R$ satisfy the sufficient conditions given in Theorem 1 to guarantee the existence and uniqueness of solution of Problem 1. If the time-step $\Delta t$ is small enough (e.g., $0<\Delta t \leq 1 / \lambda$ ), the fully-discrete Problem 4 has a unique solution $u_{h}^{n} \in$ $X_{h}$ for each $n=1, \ldots, N$.

Remark 3. As in the continuous case (see Remark 2), the fully-discrete Problem 4 allows some arbitrariness about the choice of the discrete initial data $u_{0, h}$. In fact, if $z_{h} \in X_{h} \cap$ ker $R$, we obtain the same discrete solution $u_{h}^{n}$ $(n=1, \ldots, N)$ in both cases: by taking as discrete initial data either $u_{0, h}$ or $u_{0, h}+z_{h}$.

\section{Error estimates for the fully-discrete approximation}

In this section, we will deduce some error estimates for the fully-discrete approximation. To do this, from now on we assume the assumptions of Theorems 1 and 2. Moreover, we assume that the solution to Problem 1 satisfies 
$u \in \mathrm{H}^{1}(0, T ; X)$. Furthermore, we consider the orthogonal projection operator $\Pi_{h}: X \rightarrow X_{h}$, defined by

$$
\Pi_{h} w \in X_{h}: \quad\left(\Pi_{h} w, v\right)_{X}=(w, v)_{X}, \quad \forall v \in X_{h}
$$

clearly, $\Pi_{h}$ is well-defined and satisfies

$$
\left\|w-\Pi_{h} w\right\|_{X} \leq \inf _{v \in X_{h}}\|w-v\|_{X}, \quad \forall w \in X
$$

In order to obtain the error estimates results with a regularity assumptions for the solution according to the degenerate parabolic problems, we will introduce some notations and show some relevant results.

Firstly, let us consider the Riezs isomorphism $\Lambda_{Y}: Y \rightarrow Y^{\prime}$. Then by defining $\hat{R}:=\Lambda_{Y}^{-1} R$ and following the lines of [15, Section 4], we can observe $\hat{R}: Y \rightarrow Y$ is a monotone, linear and bounded self-adjoint operator. Thus, the operator $\hat{R}$ admits a unique square root $\hat{R}^{1 / 2}$ which is also a monotone, linear and bounded self-adjoint operator (see [18]). Let $Y_{0}:=\operatorname{ker} \hat{R}, Y_{+}$be the orthogonal space of $Y_{0}$ and $Y_{+}^{1 / 2}$ be the completion of $Y_{+}$with respect the topology induced by the norm $\|v\|_{+}:=\left\|\hat{R}^{1 / 2} v\right\|_{Y}$.

We consider now the orthogonal projection operator $P_{+}: Y \rightarrow Y_{+}$defined by

$$
\hat{R} v=\hat{R}\left(P_{+} v\right), \quad \forall v \in Y .
$$

Therefore, $P_{+} \in \mathcal{L}\left(Y, Y_{+}\right)$(where $Y_{+}$is endowed with the norm of $Y$ ) and we can easily notice that

$$
\langle R v, v\rangle_{Y}=\left\|\hat{R}^{1 / 2} v\right\|_{Y}^{2}=\left\|\hat{R}^{1 / 2} P_{+} v\right\|_{Y}^{2}=\left\|P_{+} v\right\|_{+}^{2}, \quad \forall v \in Y .
$$

On the other hand, if we assume that $u \in \mathrm{H}^{1}(0, T ; X)$, then $u \in \mathrm{H}^{1}(0, T ; Y)$ and by recalling that $P_{+} \in \mathcal{L}\left(Y, Y_{+}\right)$, we obtain $P_{+} u \in \mathrm{H}^{1}\left(0, T ; Y_{+}\right) \subset \mathrm{H}^{1}(0, T ; Y)$ and $\partial_{t} P_{+} u=P_{+} \partial_{t} u$ in $\mathrm{L}^{2}\left(0, T ; Y_{+}\right)$. Hence, since $R \in \mathcal{L}\left(Y, Y^{\prime}\right)$, it follows $R P_{+} u \in \mathrm{H}^{1}\left(0, T ; Y^{\prime}\right)$ and $\partial_{t}\left(R P_{+} u\right)=R \partial_{t}\left(P_{+} u\right)$ in $\mathrm{L}^{2}\left(0, T ; Y^{\prime}\right)$. Furthermore, by noticing

$$
R v=R P_{+} v, \quad \forall v \in Y,
$$

we have $R u=R P_{+} u$ and hence $\partial_{t}(R u)=R \partial_{t}\left(P_{+} u\right)$. In summary, if $u \in$ $\mathrm{H}^{1}(0, T ; X)$ then

$$
\begin{aligned}
& P_{+} u \in \mathrm{H}^{1}\left(0, T ; Y_{+}\right) \subset \mathrm{H}^{1}(0, T ; Y), \\
& \partial_{t}(R u)=\partial_{t}\left(R P_{+} u\right)=R \partial_{t}\left(P_{+} u\right)=R P_{+} \partial_{t} u
\end{aligned}
$$

in $\mathrm{L}^{2}\left(0, T ; Y^{\prime}\right)$.

Next we want to show that the linear operator $\left.R\right|_{Y_{+}}: Y_{+} \rightarrow Y^{\prime}$ can be extended to a linear continuous operator $\tilde{R}: Y_{+}^{1 / 2} \rightarrow Y^{\prime}$. In fact, since the operator $R$ is monotone and self-adjoint, it satisfies the following Cauchy-Schwarz type inequality

$$
\left|\langle R v, w\rangle_{Y}\right| \leq\langle R v, v\rangle_{Y}^{1 / 2}\langle R w, w\rangle_{Y}^{1 / 2}, \quad \forall v, w \in Y .
$$


Consequently,

$$
\left|\langle R v, w\rangle_{Y}\right| \leq\|R\|_{\mathcal{L}\left(Y, Y^{\prime}\right)}^{\frac{1}{2}}\|v\|_{+}\|w\|_{Y}, \quad \forall v \in Y_{+}, \quad \forall w \in Y,
$$

hence

$$
\|R v\|_{Y^{\prime}} \leq\|R\|_{\mathcal{L}\left(Y, Y^{\prime}\right)}^{\frac{1}{2}}\|v\|_{+}, \quad \forall v \in Y_{+}
$$

Therefore, the restriction linear operator $\left.R\right|_{Y_{+}}: Y_{+} \rightarrow Y^{\prime}$ can be extended in an unique way to a linear continuous operator $\tilde{R}: Y_{+}^{1 / 2} \rightarrow Y^{\prime}$ by means of an standard argument of continuity and density.

Moreover, by recalling (5.4) it follows $P_{+} u \in \mathrm{H}^{1}\left(0, T ; Y_{+}\right)$and since $R$ and $\tilde{R}$ coincide in $Y_{+}$, we have $R\left(P_{+} u\right)=\tilde{R}\left(P_{+} u\right)$. Besides, since the embedding $Y_{+} \subset Y_{+}^{1 / 2}$ is continuous, we see that $P_{+} u \in \mathrm{H}^{1}\left(0, T ; Y_{+}^{1 / 2}\right)$. From this and the fact that $\tilde{R} \in \mathcal{L}\left(Y_{+}^{1 / 2}, Y^{\prime}\right)$, it follows that $R\left(P_{+} u\right)=\tilde{R}\left(P_{+} u\right) \in \mathrm{H}^{1}\left(0, T ; Y^{\prime}\right)$. Therefore, if $P_{+} u \in \mathcal{C}^{1}\left([0, T] ; Y_{+}^{1 / 2}\right)$ then

$$
\begin{aligned}
& R\left(P_{+} u\right)=\tilde{R}\left(P_{+} u\right) \quad \text { in } \mathcal{C}^{1}\left([0, T] ; Y^{\prime}\right), \\
& \partial_{t}\left(R P_{+} u\right)=\tilde{R} \partial_{t}\left(P_{+} u\right) \quad \text { in } \mathcal{C}^{0}\left([0, T] ; Y^{\prime}\right) \subset \mathcal{C}^{0}\left([0, T] ; X^{\prime}\right)
\end{aligned}
$$

Consequently, by assuming that the solution of Problem 1 satisfies $u \in$ $\mathrm{H}^{1}(0, T ; X)$ and $P_{+} u \in \mathcal{C}^{1}\left([0, T] ; Y_{+}^{1 / 2}\right)$, from (5.5) and (5.7) it follows

$$
\partial_{t}(R u)=\partial_{t}\left(R P_{+} u\right)=\tilde{R} \partial_{t}\left(P_{+} u\right) \quad \text { in } \mathcal{C}^{0}\left([0, T] ; Y^{\prime}\right) .
$$

Hence, the first equation of Problem 1 implies

$$
\left\langle\tilde{R}\left(\partial_{t}\left(P_{+} u\right)(t)\right), v\right\rangle_{Y}+\langle A(t) u(t), v\rangle_{X}=\langle f(t), v\rangle_{X}, \forall v \in X, \forall t \in[0, T] .
$$

Furthermore, by using (5.3) the first equation of Problem 4 yields

$$
\left\langle\tilde{R}\left(\bar{\partial}\left(P_{+} u_{h}^{n}\right)\right), v\right\rangle_{Y}+\left\langle A\left(t_{n}\right) u_{h}^{n}, v\right\rangle_{X}=\langle f(t), v\rangle_{X}, \quad \forall v \in X_{h}
$$

for $n=1, \ldots, N$.

Now, we introduce the standard terms to obtain the error estimates for parabolic problems. To this aim, from now on $u$ and $u_{h}^{n}(n=1, \ldots, N)$ denote the solutions of Problem 1 and Problem 4, respectively. We define the error and consider its splitting

$$
e_{h}^{n}:=u\left(t_{n}\right)-u_{h}^{n}=\rho_{h}^{n}+\sigma_{h}^{n}, \quad n=0, \ldots, N,
$$

where

$$
\rho_{h}(t):=u(t)-\Pi_{h} u(t), \quad \rho_{h}^{n}:=\rho_{h}\left(t_{n}\right), \quad \sigma_{h}^{n}:=\Pi_{h} u\left(t_{n}\right)-u_{h}^{n} .
$$

Finally, by assuming $P_{+} u \in \mathcal{C}^{1}\left([0, T] ; Y_{+}^{1 / 2}\right)$ (see (5.2)) we denote

$$
\tau^{n}:=\frac{P_{+} u\left(t_{n}\right)-P_{+} u\left(t_{n-1}\right)}{\Delta t}-\partial_{t} P_{+} u\left(t_{n}\right)=\bar{\partial}\left(P_{+} u\left(t_{n}\right)\right)-\partial_{t} P_{+} u\left(t_{n}\right),
$$


for $n=1, \ldots, N$. Note that $\tau^{n} \in Y_{+}^{1 / 2}$, but in general $\tau^{n} \notin Y$. Moreover, we can easily check that

$$
\partial_{t} P_{+} u\left(t_{n}\right)-\bar{\partial}\left(P_{+} u_{h}^{n}\right)=\bar{\partial}\left(P_{+} e_{h}^{n}\right)-\tau^{n}=\bar{\partial}\left(P_{+} \rho_{h}^{n}\right)+\bar{\partial}\left(P_{+} \sigma_{h}^{n}\right)-\tau^{n}
$$

for $n=1, \ldots, N$.

Lemma 1. If $u \in \mathrm{H}^{1}(0, T ; X)$ with $P_{+} u \in \mathcal{C}^{1}\left([0, T] ; Y_{+}^{1 / 2}\right)$ then there exists a constant $C>0$, independent of $h$ and $\Delta t$, such that

$$
\begin{aligned}
& \left\langle R \sigma_{h}^{n}, \sigma_{h}^{n}\right\rangle_{Y}+\Delta t \sum_{k=1}^{n}\left\|\sigma_{h}^{k}\right\|_{X}^{2} \\
& \leq C\left[\left\langle R \sigma_{h}^{0}, \sigma_{h}^{0}\right\rangle_{Y}+\Delta t \sum_{k=1}^{N}\left\{\left\|\tau^{k}\right\|_{+}^{2}+\left\|\bar{\partial} \rho_{h}^{k}\right\|_{X}^{2}+\left\|\rho_{h}^{k}\right\|_{X}^{2}\right\}\right] .
\end{aligned}
$$

Furthermore, if $u_{0} \in X$ and for each $t \in[0, T]$ the operator $A(t)$ is monotone and there exists a constant $C>0$ such that

$$
\left\langle A^{\prime}(t) w, v\right\rangle \leq C\|w\|_{X}\|v\|_{X}, \quad \forall w, v \in X, \quad \forall t \in[0, T]
$$

then, there exists a constant $C>0$, independent of $h$ and $\Delta t$, such that

$$
\begin{aligned}
& \Delta t \sum_{k=1}^{n}\left\langle R \bar{\partial} \sigma_{h}^{k}, \bar{\partial} \sigma_{h}^{k}\right\rangle_{Y}+\left\langle A\left(t_{n}\right) \sigma_{h}^{n}, \sigma_{h}^{n}\right\rangle_{X} \\
& \leq C\left[\left\|\sigma_{h}^{0}\right\|_{X}^{2}+\left\|\rho_{h}^{0}\right\|_{X}^{2}+\left\|\rho_{h}^{n}\right\|_{X}^{2}+\Delta t \sum_{k=1}^{N}\left\{\left\|\tau^{k}\right\|_{+}^{2}+\left\|\bar{\partial} \rho_{h}^{k}\right\|_{X}^{2}+\left\|\rho_{h}^{k}\right\|_{X}^{2}\right\}\right] .
\end{aligned}
$$

Proof. $\quad$ Let $n \in\{1, \ldots, N\}, k \in\{1, \ldots, n\}$ and $v \in X_{h}$. From (5.8) and (5.9) it follows

$$
\left\langle\tilde{R}\left(\partial_{t}\left(P_{+} u\right)\left(t_{k}\right)-\bar{\partial}\left(P_{+} u_{h}^{k}\right)\right), v\right\rangle_{Y}+\left\langle A\left(t_{k}\right) e_{h}^{k}, v\right\rangle_{X}=0, \quad \forall v \in X_{h},
$$

for $k=1, \ldots, n$. Then, using (5.13) we obtain

$$
\begin{aligned}
& \left\langle\tilde{R} \bar{\partial}\left(P_{+} \sigma_{h}^{k}\right), v\right\rangle_{Y}+\left\langle A\left(t_{k}\right) \sigma_{h}^{k}, v\right\rangle_{X} \\
& =\left\langle\tilde{R} \tau^{k}, v\right\rangle_{Y}-\left\langle\tilde{R} \bar{\partial}\left(P_{+} \rho_{h}^{k}\right), v\right\rangle_{Y}-\left\langle A\left(t_{k}\right) \rho_{h}^{k}, v\right\rangle_{X}, \quad \forall v \in X_{h} .
\end{aligned}
$$

Therefore, by recalling $\tilde{R}\left(P_{+} v\right)=R\left(P_{+} v\right)=R v$ for all $v \in Y$, it follows

$$
\begin{aligned}
& \left\langle R \bar{\partial} \sigma_{h}^{k}, v\right\rangle_{Y}+\left\langle A\left(t_{k}\right) \sigma_{h}^{k}, v\right\rangle_{X} \\
& =\left\langle\tilde{R} \tau^{k}, v\right\rangle_{Y}-\left\langle R \bar{\partial} \rho_{h}^{k}, v\right\rangle_{Y}-\left\langle A\left(t_{k}\right) \rho_{h}^{k}, v\right\rangle_{X}, \quad \forall v \in X_{h} .
\end{aligned}
$$

By testing this previous identity with $v=\sigma_{h}^{k} \in X_{h}$, we have

$$
\begin{aligned}
& \left\langle R \bar{\partial} \sigma_{h}^{k}, \sigma_{h}^{k}\right\rangle_{Y}+\left\langle A\left(t_{k}\right) \sigma_{h}^{k}, \sigma_{h}^{k}\right\rangle_{X} \\
& =\left\langle\tilde{R} \tau^{k}, \sigma_{h}^{k}\right\rangle_{Y}-\left\langle R \bar{\partial} \rho_{h}^{k}, \sigma_{h}^{k}\right\rangle_{Y}-\left\langle A\left(t_{k}\right) \rho_{h}^{k}, \sigma_{h}^{k}\right\rangle_{X} .
\end{aligned}
$$


Using the fact that $R$ is monotone and self-adjoint, the first term of the lefthand term in the previous identity satisfies

$$
\left\langle R \bar{\partial} \sigma_{h}^{k}, \sigma_{h}^{k}\right\rangle_{Y} \geq \frac{1}{2 \Delta t}\left[\left\langle R \sigma_{h}^{k}, \sigma_{h}^{k}\right\rangle_{Y}-\left\langle R \sigma_{h}^{k-1}, \sigma_{h}^{k-1}\right\rangle_{Y}\right]
$$

by recalling (3.1), there exist $\lambda, \alpha>0$ such that

$$
\left\langle A\left(t_{k}\right) \sigma_{h}^{k}, \sigma_{h}^{k}\right\rangle_{X} \geq \alpha\left\|\sigma_{h}^{k}\right\|_{X}^{2}-\lambda\left\langle R \sigma_{h}^{k}, \sigma_{h}^{k}\right\rangle_{Y},
$$

thus, replacing in (5.18), it follows that

$$
\begin{aligned}
& \frac{1}{2 \Delta t}\left[\left\langle R \sigma_{h}^{k}, \sigma_{h}^{k}\right\rangle_{Y}-\left\langle R \sigma_{h}^{k-1}, \sigma_{h}^{k-1}\right\rangle_{Y}\right]+\alpha\left\|\sigma_{h}^{k}\right\|_{X}^{2}-\lambda\left\langle R \sigma_{h}^{k}, \sigma_{h}^{k}\right\rangle_{Y} \\
& \leq\left\langle\tilde{R} \tau^{k}, \sigma_{h}^{k}\right\rangle_{Y}-\left\langle R \bar{\partial} \rho_{h}^{k}, \sigma_{h}^{k}\right\rangle_{Y}-\left\langle A\left(t_{k}\right) \rho_{h}^{k}, \sigma_{h}^{k}\right\rangle_{X} .
\end{aligned}
$$

Now, from inequality (5.6) we can deduce the following inequality by using an standard argument of continuity and density

$$
\left|\langle\tilde{R} v, w\rangle_{Y}\right| \leq\|v\|_{+}\langle R w, w\rangle_{Y}^{1 / 2}, \quad \forall v \in Y_{+}^{1 / 2}, \quad \forall w \in Y
$$

hence, by recalling that $\tau^{k} \in Y_{+}^{1 / 2}$ (see (5.12)), we obtain

$$
\left|\left\langle\tilde{R} \tau^{k}, \sigma_{h}^{k}\right\rangle_{Y}\right| \leq \frac{1}{4}\left\langle R \sigma_{h}^{k}, \sigma_{h}^{k}\right\rangle_{Y}+\left\|\tau^{k}\right\|_{+}^{2} .
$$

Besides, from (5.6) we have

$$
\left|\left\langle R \bar{\partial} \rho_{h}^{k}, \sigma_{h}^{k}\right\rangle_{Y}\right| \leq \frac{1}{4}\left\langle R \sigma_{h}^{k}, \sigma_{h}^{k}\right\rangle_{Y}+\left\langle R \bar{\partial} \rho_{h}^{k}, \bar{\partial} \rho_{h}^{k}\right\rangle_{Y}
$$

On the other hand, by using the uniform continuity of the family of operators $A(t)$, we can notice that

$$
\left|\left\langle A\left(t_{k}\right) \rho_{h}^{k}, \sigma_{h}^{k}\right\rangle_{X}\right| \leq \frac{\alpha}{2}\left\|\sigma_{h}^{k}\right\|_{X}^{2}+\frac{1}{2 \alpha} M^{2}\left\|\rho_{h}^{k}\right\|_{X}^{2} .
$$

Therefore, by replacing (5.20)-(5.21) in (5.19) and using the fact that $R$ is a bounded operator and $X \subset Y$ is a continuous embedding, we deduce

$$
\begin{aligned}
& \left\langle R \sigma_{h}^{k}, \sigma_{h}^{k}\right\rangle_{Y}-\left\langle R \sigma_{h}^{k-1}, \sigma_{h}^{k-1}\right\rangle_{Y}+\alpha \Delta t\left\|\sigma_{h}^{k}\right\|_{X}^{2} \\
& \leq(1+2 \lambda) \Delta t\left\langle R \sigma_{h}^{k}, \sigma_{h}^{k}\right\rangle_{Y}+C \Delta t\left\{\left\|\tau^{k}\right\|_{+}^{2}+\left\|\bar{\partial} \rho_{h}^{k}\right\|_{X}^{2}+\left\|\rho_{h}^{k}\right\|_{X}^{2}\right\} .
\end{aligned}
$$

Hence, by summing over $k$, we obtain

$$
\begin{aligned}
& \left\langle R \sigma_{h}^{n}, \sigma_{h}^{n}\right\rangle_{Y}-\left\langle R \sigma_{h}^{0}, \sigma_{h}^{0}\right\rangle_{Y}+\alpha \Delta t \sum_{k=1}^{n}\left\|\sigma_{h}^{k}\right\|_{X}^{2} \\
& \leq(1+2 \lambda) \Delta t \sum_{k=1}^{n}\left\langle R \sigma_{h}^{k}, \sigma_{h}^{k}\right\rangle_{Y}+C \Delta t \sum_{k=1}^{n}\left\{\left\|\tau^{k}\right\|_{+}^{2}+\left\|\bar{\partial} \rho_{h}^{k}\right\|_{X}^{2}+\left\|\rho_{h}^{k}\right\|_{X}^{2}\right\} .
\end{aligned}
$$


Then, if $\Delta t$ is small enough such that $(1+2 \lambda) \Delta t \leq \frac{1}{2}$, we have

$$
\begin{aligned}
& \frac{1}{2}\left\langle R \sigma_{h}^{n}, \sigma_{h}^{n}\right\rangle_{Y}+\alpha \Delta t \sum_{k=1}^{n}\left\|\sigma_{h}^{k}\right\|_{X}^{2} \leq(1+2 \lambda) \Delta t \sum_{k=1}^{n-1}\left\langle R \sigma_{h}^{k}, \sigma_{h}^{k}\right\rangle_{Y} \\
& +\left\langle R \sigma_{h}^{0}, \sigma_{h}^{0}\right\rangle_{Y}+C \Delta t \sum_{k=1}^{n}\left\{\left\|\tau^{k}\right\|_{+}^{2}+\left\|\bar{\partial} \rho_{h}^{k}\right\|_{X}^{2}+\left\|\rho_{h}^{k}\right\|_{X}^{2}\right\},
\end{aligned}
$$

which implies

$$
\begin{aligned}
& \left\langle R \sigma_{h}^{n}, \sigma_{h}^{n}\right\rangle_{Y} \leq 2\left\langle R \sigma_{h}^{0}, \sigma_{h}^{0}\right\rangle_{Y} \\
& +2(1+2 \lambda) \Delta t \sum_{k=1}^{n-1}\left\langle R \sigma_{h}^{k}, \sigma_{h}^{k}\right\rangle_{Y}+C \Delta t \sum_{k=1}^{n}\left[\left\|\tau^{k}\right\|_{+}^{2}+\left\|\bar{\partial} \rho_{h}^{k}\right\|_{X}^{2}+\left\|\rho_{h}^{k}\right\|_{X}^{2}\right] .
\end{aligned}
$$

Therefore, by using the discrete Gronwall's Lemma (see, for instance, [17, Lemma 1.4.2]), we obtain for $n \in\{1, \ldots, N\}$

$$
\left\langle R \sigma_{h}^{n}, \sigma_{h}^{n}\right\rangle_{Y} \leq C\left[\left\langle R \sigma_{h}^{0}, \sigma_{h}^{0}\right\rangle_{Y}+\Delta t \sum_{k=1}^{n}\left\{\left\|\tau^{k}\right\|_{+}^{2}+\left\|\bar{\partial} \rho_{h}^{k}\right\|_{X}^{2}+\left\|\rho_{h}^{k}\right\|_{X}^{2}\right\}\right] .
$$

Hence, by using this inequality to estimate the first term in the right-hand term of $(5.22)$, we deduce (5.14).

Next, we want to prove (5.16) by assuming that each $A(t)$ is monotone and (5.15) holds true. In fact, by taking $v=\bar{\partial} \sigma_{h}^{k} \in X_{h}$ in (5.17), we obtain

$$
\begin{aligned}
& \left\langle R \bar{\partial} \sigma_{h}^{k}, \bar{\partial} \sigma_{h}^{k}\right\rangle_{Y}+\left\langle A\left(t_{k}\right) \sigma_{h}^{k}, \bar{\partial} \sigma_{h}^{k}\right\rangle_{X} \\
& =\left\langle\tilde{R} \tau^{k}, \bar{\partial} \sigma_{h}^{k}\right\rangle_{Y}-\left\langle R \bar{\partial} \rho_{h}^{k}, \bar{\partial} \sigma_{h}^{k}\right\rangle_{Y}-\left\langle A\left(t_{k}\right) \rho_{h}^{k}, \bar{\partial} \sigma_{h}^{k}\right\rangle_{X} .
\end{aligned}
$$

Now, since each operator $A(t)$ is monotone and self-adjoint, it follows

$$
\left\langle A\left(t_{k}\right) \sigma_{h}^{k}, \bar{\partial} \sigma_{h}^{k}\right\rangle_{X} \geq \frac{1}{2 \Delta t}\left\{\left\langle A\left(t_{k}\right) \sigma_{h}^{k}, \sigma_{h}^{k}\right\rangle_{X}-\left\langle A\left(t_{k}\right) \sigma_{h}^{k-1}, \sigma_{h}^{k-1}\right\rangle_{X}\right\}
$$

and therefore

$$
\begin{aligned}
\left\langle A\left(t_{k}\right) \sigma_{h}^{k}, \bar{\partial} \sigma_{h}^{k}\right\rangle_{X} \geq & \frac{1}{2 \Delta t}\left[\left\langle A\left(t_{k}\right) \sigma_{h}^{k}, \sigma_{h}^{k}\right\rangle_{X}-\left\langle A\left(t_{k-1}\right) \sigma_{h}^{k-1}, \sigma_{h}^{k-1}\right\rangle_{X}\right] \\
& -\frac{1}{2 \Delta t}\left\langle\left(\int_{t_{k-1}}^{t_{k}} A^{\prime}(t) d t\right) \sigma_{h}^{k-1}, \sigma_{h}^{k-1}\right\rangle_{X} .
\end{aligned}
$$

On the other hand, a straightforward computation shows that

$$
\begin{aligned}
& \left\langle A\left(t_{k}\right) \rho_{h}^{k}, \bar{\partial} \sigma_{h}^{k}\right\rangle_{X}=\frac{1}{\Delta t}\left[\left\langle A\left(t_{k}\right) \rho_{h}^{k}, \sigma_{h}^{k}\right\rangle_{X}-\left\langle A\left(t_{k-1}\right) \rho_{h}^{k-1}, \sigma_{h}^{k-1}\right\rangle_{X}\right] \\
& -\left\langle A\left(t_{k}\right) \bar{\partial} \rho_{h}^{k}, \sigma_{h}^{k-1}\right\rangle_{X}-\frac{1}{\Delta t}\left\langle\left(\int_{t_{k-1}}^{t_{k}} A^{\prime}(t) d t\right) \rho_{h}^{k-1}, \sigma_{h}^{k-1}\right\rangle_{X} .
\end{aligned}
$$


Hence, by using (5.24) and (5.25) in (5.23), we have

$$
\begin{aligned}
& \left\langle R \bar{\partial} \sigma_{h}^{k}, \bar{\partial} \sigma_{h}^{k}\right\rangle_{Y}+\frac{1}{2 \Delta t}\left[\left\langle A\left(t_{k}\right) \sigma_{h}^{k}, \sigma_{h}^{k}\right\rangle_{X}-\left\langle A\left(t_{k-1}\right) \sigma_{h}^{k-1}, \sigma_{h}^{k-1}\right\rangle_{X}\right] \\
& \leq\left\langle A\left(t_{k}\right) \bar{\partial} \rho_{h}^{k}, \sigma_{h}^{k-1}\right\rangle_{X}+\frac{1}{\Delta t}\left\langle\left(\int_{t_{k-1}}^{t_{k}} A^{\prime}(t) d t\right)\left(\rho_{h}^{k-1}+\frac{1}{2} \sigma_{h}^{k-1}\right), \sigma_{h}^{k-1}\right\rangle_{X} \\
& +\left\langle\tilde{R} \tau^{k}, \bar{\partial} \sigma_{h}^{k}\right\rangle_{Y}-\left\langle R \bar{\partial} \rho_{h}^{k}, \bar{\partial} \sigma_{h}^{k}\right\rangle_{Y}-\frac{1}{\Delta t}\left[\left\langle A\left(t_{k}\right) \rho_{h}^{k}, \sigma_{h}^{k}\right\rangle_{X}-\left\langle A\left(t_{k-1}\right) \rho_{h}^{k-1}, \sigma_{h}^{k-1}\right\rangle_{X}\right]
\end{aligned}
$$

then, recalling that the family of operators $A(t)$ is uniformly bounded and that the operator $R$ is also bounded, using (5.6), (5.20), (5.15) and $X \subset Y$ is a continuous embedding, it follows that

$$
\begin{aligned}
\frac{1}{2}\left\langle R \bar{\partial} \sigma_{h}^{k}, \bar{\partial} \sigma_{h}^{k}\right\rangle_{Y}+\frac{1}{2 \Delta t}\left[\left\langle A\left(t_{k}\right) \sigma_{h}^{k}, \sigma_{h}^{k}\right\rangle_{X}-\left\langle A\left(t_{k-1}\right) \sigma_{h}^{k-1}, \sigma_{h}^{k-1}\right\rangle_{X}\right] \\
\leq-\frac{1}{\Delta t}\left[\left\langle A\left(t_{k}\right) \rho_{h}^{k}, \sigma_{h}^{k}\right\rangle_{X}-\left\langle A\left(t_{k-1}\right) \rho_{h}^{k-1}, \sigma_{h}^{k-1}\right\rangle_{X}\right] \\
+C\left[\left\|\sigma_{h}^{k-1}\right\|_{X}^{2}+\left\|\tau^{k}\right\|_{+}^{2}+\left\|\bar{\partial} \rho_{h}^{k}\right\|_{X}^{2}+\left\|\rho_{h}^{k-1}\right\|_{X}^{2}\right]
\end{aligned}
$$

then, multiplying by $2 \Delta t$, summing over $k$ and using the fact that

$$
\left|\left\langle A\left(t_{n}\right) \rho_{h}^{n}, \sigma_{h}^{n}\right\rangle_{X}\right| \leq\left\langle A\left(t_{n}\right) \rho_{h}^{n}, \rho_{h}^{n}\right\rangle_{X}+\frac{1}{4}\left\langle A\left(t_{n}\right) \sigma_{h}^{n}, \sigma_{h}^{n}\right\rangle_{X},
$$

we obtain

$$
\begin{aligned}
& \Delta t \sum_{k=1}^{n}\left\langle R \bar{\partial} \sigma_{h}^{k}, \bar{\partial} \sigma_{h}^{k}\right\rangle_{Y}+\frac{1}{2}\left\langle A\left(t_{n}\right) \sigma_{h}^{n}, \sigma_{h}^{n}\right\rangle_{X} \leq\left\langle A(0)\left(2 \rho_{h}^{0}+\sigma_{h}^{0}\right), \sigma_{h}^{0}\right\rangle_{X} \\
& \quad+2\left\langle A\left(t_{n}\right) \rho_{h}^{n}, \rho_{h}^{n}\right\rangle_{X}+C \Delta t \sum_{k=1}^{n}\left\{\left\|\sigma_{h}^{k-1}\right\|_{X}^{2}+\left\|\tau^{k}\right\|_{+}^{2}+\left\|\bar{\partial} \rho_{h}^{k}\right\|_{X}^{2}+\left\|\rho_{h}^{k-1}\right\|_{X}^{2}\right\} .
\end{aligned}
$$

Finally, using (5.14) to estimate the sum involving $\left\|\sigma_{h}^{k-1}\right\|_{X}$ and recalling $A(t)$ is uniformly bounded and monotone, we deduce (5.16).

Our next goal is to prove the error estimates.

Theorem 3. If $u \in \mathrm{H}^{1}(0, T ; X)$ with $P_{+} u \in H^{2}\left(0, T ; Y_{+}^{1 / 2}\right)$, then there exists a constant $C>0$, independent of $h$ and $\Delta t$, such that

$$
\begin{aligned}
& \max _{1 \leq n \leq N}\left\langle R\left(u\left(t_{n}\right)-u_{h}^{n}\right), u\left(t_{n}\right)-u_{h}^{n}\right\rangle_{Y}+\Delta t \sum_{n=1}^{N}\left\|u\left(t_{n}\right)-u_{h}^{n}\right\|_{X}^{2} \\
\leq C & \left\{R\left(u_{0}-u_{0, h}\right), u_{0}-u_{0, h}\right\rangle_{Y}+\max _{0 \leq n \leq N}\left[\inf _{v \in X_{h}}\left\|u\left(t_{n}\right)-v\right\|_{X}^{2}\right] \\
& \left.+\int_{0}^{T} \inf _{v \in X_{h}}\left\|\partial_{t} u(t)-v\right\|_{X}^{2} d t+(\Delta t)^{2} \int_{0}^{T}\left\|\partial_{t t} P_{+} u(t)\right\|_{+}^{2} d t\right\} .
\end{aligned}
$$


Furthermore, if for each $t \in[0, T]$ the operator $A(t)$ is monotone and (5.15) holds true, then there exists a constant $C>0$ independent of $h$ and $\Delta t$, satisfying

$$
\begin{aligned}
& \Delta t \sum_{k=1}^{N}\left\|\partial_{t}\left(P_{+} u\right)\left(t_{k}\right)-\bar{\partial} P_{+} u_{h}^{k}\right\|_{+}^{2}+\max _{1 \leq n \leq N}\left\langle A\left(t_{n}\right)\left(u\left(t_{n}\right)-u_{h}^{n}\right), u\left(t_{n}\right)-u_{h}^{n}\right\rangle_{X} \\
& \leq C\left\{\left\|u(0)-u_{0, h}\right\|_{X}^{2}+\max _{0 \leq n \leq N}\left[\inf _{v \in X_{h}}\left\|u\left(t_{n}\right)-v\right\|_{X}^{2}\right]\right. \\
& \left.\quad+\int_{0}^{T} \inf _{v \in X_{h}}\left\|\partial_{t} u(t)-v\right\|_{X}^{2} d t+(\Delta t)^{2} \int_{0}^{T}\left\|\partial_{t t} P_{+} u(t)\right\|_{+}^{2} d t\right\}
\end{aligned}
$$

Remark 4. The first term on the right hand side of (5.26) is

$$
\left\langle R\left(u_{0}-u_{0, h}\right), u_{0}-u_{0, h}\right\rangle_{Y} .
$$

We can easily check that this term does not change if $u_{0}$ and $u_{0, h}$ are respectively replaced with $u_{0}+z$ and $u_{0, h}+z_{h}$ for any $z \in X \cap \operatorname{ker} R$ and any $z_{h} \in X_{h} \cap \operatorname{ker} R$, respectively. This property is very convenient in view of the "freedom of choosing" the continuous and the discrete initial data (cf. Remark 2 and Remark 3).

Remark 5. The initial condition of the problem is $R u(0)=R u_{0}$, then $u(0)$ and $u_{0}$ can be different. Therefore, the term $\left\|u(0)-u_{0, h}\right\|_{X}$ which appears on the right hand side of (5.27) is not an actual a priori error estimate, because $u(0)$ is not a data of the problem. At the end of this section, we will show some conditions to bound this norm in terms of the initial data error $\left\|u_{0}-u_{0, h}\right\|_{X}$.

Proof. First of all, we notice that (5.11) and (5.1) imply

$$
\left\|\rho_{h}^{n}\right\|_{X}=\left\|\rho_{h}\left(t_{n}\right)\right\|_{X} \leq C \inf _{z \in X_{h}}\left\|u\left(t_{n}\right)-z\right\|_{X}
$$

Moreover, the regularity assumption about $u$ implies $\partial_{t} \Pi_{h} u(\cdot)=\Pi_{h}\left(\partial_{t} u(\cdot)\right)$ and consequently

$$
\int_{0}^{T}\left\|\partial_{t} \rho_{h}(t)\right\|_{X} d t \leq C \int_{0}^{T} \inf _{z \in X_{h}}\left\|\partial_{t} u(t)-z\right\|_{X} d t
$$

Hence, it is easy to check that

$$
\begin{aligned}
\Delta t \sum_{k=1}^{N}\left\|\bar{\partial} \rho_{h}^{k}\right\|_{X}^{2} & =\Delta t \sum_{k=1}^{N}\left\|\frac{1}{\Delta t} \int_{t_{k-1}}^{t_{k}} \partial_{t} \rho_{h}(t) d t\right\|_{X}^{2} \\
& \leq \sum_{k=1}^{N} \int_{t_{k-1}}^{t_{k}}\left\|\partial_{t} \rho_{h}(t)\right\|_{X}^{2} d t \leq C \int_{0}^{T} \inf _{v \in X_{h}}\left\|\partial_{t} u(t)-v\right\|_{X}^{2} d t .
\end{aligned}
$$

On the other hand, by combining a Taylor expansion with the CauchySchwarz inequality, we obtain

$$
\sum_{k=1}^{N}\left\|\tau^{k}\right\|_{+}^{2}=\sum_{k=1}^{N}\left\|\frac{1}{\Delta t} \int_{t_{k-1}}^{t_{k}}\left(t_{k-1}-t\right) \partial_{t t} P_{+} u(t) d t\right\|_{+}^{2} \leq \Delta t \int_{0}^{T}\left\|\partial_{t t} P_{+} u(t)\right\|_{+}^{2} d t
$$


Now, by writing $\sigma_{h}^{0}=e_{h}^{0}-\rho_{h}^{0}$ and using the fact that $R$ is self-adjoint and monotone $^{1}$, from the second equation of Problem 1 , it follows that

$$
\left\langle R \sigma_{h}^{0}, \sigma_{h}^{0}\right\rangle_{Y} \leq 2\left\langle R\left(u_{0}-u_{0, h}\right), u_{0}-u_{0, h}\right\rangle_{Y}+2\left\langle R \rho_{h}^{0}, \rho_{h}^{0}\right\rangle_{Y}
$$

By using inequalities (5.28)-(5.29) and Lemma 1, (5.26) follows from the fact that $u\left(t_{n}\right)-u_{h}^{n}=\rho_{h}^{n}+\sigma_{h}^{n}$ (see (5.10)) and the triangle inequality.

Next, we need to deduce (5.27). From (5.13) it follows that

$$
\left\|\partial_{t}\left(P_{+} u\right)\left(t_{k}\right)-\bar{\partial} P_{+} u_{h}^{k}\right\|_{+}^{2} \leq C\left[\left\langle R \bar{\partial} \sigma_{h}^{k}, \bar{\partial} \sigma_{h}^{k}\right\rangle_{Y}+\left\|\bar{\partial} \rho_{h}^{k}\right\|_{Y}^{2}+\left\|\tau^{k}\right\|_{+}^{2}\right] .
$$

Consequently, (5.27) follows by using (5.16), by proceeding as in the proof of (5.26) and noticing that

$$
\begin{aligned}
& \left\|\sigma_{h}^{0}\right\|_{X}^{2} \leq 2\left\|u(0)-u_{0, h}\right\|_{X}^{2}+2\left\|\rho_{h}^{0}\right\|_{X}^{2} \\
& \Delta t \sum_{n=1}^{N} \inf _{v \in X_{h}}\left\|u\left(t_{n}\right)-v\right\|_{X}^{2} \leq T \max _{1 \leq n \leq N}\left[\inf _{v \in X_{h}}\left\|u\left(t_{n}\right)-v\right\|_{X}^{2}\right] .
\end{aligned}
$$

Remark 6 . To end this section, we will discuss around the term $\left\|u(0)-u_{0, h}\right\|_{X}$ which appears in the estimate (5.27) (see Remark 5). We start by proving that under some assumptions about the solution and the data of the problem, $u(0)$ is uniquely determined by $R u_{0}$ and $f$.

Let $X_{0}:=X \cap \operatorname{ker} R$ and assume that $f \in \mathcal{C}^{0}\left([0, T] ; X^{\prime}\right)$. By using (3.1), we can easily check that for any $v \in X$ there exists a unique $\mathcal{E} v \in X$ such that:

$$
\mathcal{E} v-v \in X_{0}, \quad\langle A(0) \mathcal{E} v, w\rangle_{X}=\langle f(0), w\rangle_{X}, \quad \forall w \in X_{0} .
$$

The operator $\mathcal{E}: X \rightarrow X$ satisfies $\mathcal{E}^{2}=\mathcal{E}$. Moreover, if the initial data $u_{0} \in X$ and the solution $u$ of Problem 1 satisfies $u \in \mathcal{C}^{0}([0, T] ; X)$ then $u(0)=\mathcal{E} u_{0}$. Consequently, if $u_{0} \in X$ and the assumptions of Theorem 3 hold true, then

$$
\left\|u(0)-u_{0, h}\right\|_{X}=\left\|\mathcal{E} u_{0}-u_{0, h}\right\|_{X} .
$$

In the same manner we can define a discrete version of the operator $\mathcal{E}$. In fact, if we denote $X_{0, h}:=X_{h} \cap \operatorname{ker} R$, the operator $\mathcal{E}_{h}: X_{h} \rightarrow X_{h}$ characterized by (for any $v_{h} \in X_{h}$ ):

$$
\mathcal{E}_{h} v_{h}-v_{h} \in X_{0, h} \quad\left\langle A(0) \mathcal{E}_{h} v_{h}, w_{h}\right\rangle_{X}=\left\langle f(0), w_{h}\right\rangle_{X}, \quad \forall w_{h} \in X_{0, h}
$$

is well defined and satisfies $\mathcal{E}_{h}^{2}=\mathcal{E}_{h}$. Furthermore, for any $v \in X$ and $v_{h} \in X_{h}$ the following estimate holds true for some $C>0$ independent of $h$ :

$$
\left\|\mathcal{E} v-\mathcal{E}_{h} v_{h}\right\|_{X} \leq C\left[\left\|v-v_{h}\right\|_{X}+\inf _{w_{h} \in X_{0, h}}\left\|(\mathcal{E} v-v)-w_{h}\right\|_{X}\right]
$$

\footnotetext{
${ }^{1}$ Notice that if $R$ is self-adjoint and monotone, we have $\langle R(v+w),(v+w)\rangle_{Y} \leq$ $2\left[\langle R(v), v\rangle_{Y}+\langle R(w), w\rangle_{Y}\right]$ for any $v, w \in Y$.
} 
On the other hand, by testing the first equation of Problem 4 with $w_{h} \in X_{0, h}$ it follows

$$
\left\langle A\left(t_{n}\right) u_{h}^{n}, w_{h}\right\rangle_{X}=\left\langle f\left(t_{n}\right), w_{h}\right\rangle_{X}, \quad \forall w_{h} \in X_{0, h}
$$

for $n=1, \ldots, N$. Therefore, then we could choice $u_{0, h} \in X_{h}$ such that

$$
\left\langle A(0) u_{0, h}, w_{h}\right\rangle_{X}=\left\langle f(0), w_{h}\right\rangle_{X}, \quad \forall w_{h} \in X_{0, h},
$$

which implies $u_{0, h}=\mathcal{E}_{h} u_{0 . h}$. Hence, by using (5.31) and (5.33) we obtain

$$
\begin{aligned}
\left\|u(0)-u_{0, h}\right\|_{X} & =\left\|\mathcal{E} u_{0}-\mathcal{E}_{h} u_{0, h}\right\|_{X} \\
& \leq C\left[\left\|u_{0}-u_{0, h}\right\|_{X}+\inf _{w_{h} \in X_{0, h}}\left\|\left(\mathcal{E} u_{0}-u_{0}\right)-w_{h}\right\|_{X}\right] .
\end{aligned}
$$

This estimate shows that if $\mathcal{E} u_{0}-u_{0} \in X_{0}$ can be satisfactorily approximated in $X_{0, h}$, then $\left\|u(0)-u_{0, h}\right\|_{X}$ goes to zero if $h \rightarrow 0$ when the initial error $\left\|u_{0}-u_{0, h}\right\|_{X}$ goes to zero. Moreover, if Problem 1 is equivalently formulated by considering the new initial data $\hat{u}_{0}:=u_{0}+z$ with $z:=\mathcal{E} u_{0}-u_{0} \in X_{0}$ (cf. Remark 2), then the new initial data $\hat{u}_{0}$ satisfies $\hat{u}_{0}=\mathcal{E} u_{0}=u(0)$ and therefore from (5.34) it follows

$$
\left\|u(0)-u_{0, h}\right\|_{X}=\left\|\hat{u}_{0}-u_{0, h}\right\|_{X}
$$

\section{Application to the eddy current problem}

The eddy current model is obtained by dropping the displacement currents from Maxwell equations [7, Chapter 8]) and it provides a reasonable approximation to the solution of the full Maxwell system in the low frequency range (see [2]). This model is commonly used in many problems in science and industry: induction heating, electromagnetic braking, electric generation, etc (see [1, Chapter 9]). The purpose for the eddy current problem is to determine the eddy currents induced a three-dimensional conducting domain $\hat{\Omega}_{\mathrm{C}}$ by a given time dependent compactly-supported current density $\mathbf{J}$. The eddy current problem can be read as follows.

Problem 5. Find the magnetic field $\mathbf{H}: \mathbb{R}^{3} \times[0, T] \rightarrow \mathbb{R}^{3}$ and the electric field $\mathbf{E}: \mathbb{R}^{3} \times[0, T] \rightarrow \mathbb{R}^{3}$ satisfying

$$
\begin{aligned}
& \partial_{t}(\mu \mathbf{H})+\operatorname{curl} \mathbf{E}=\mathbf{0}, \quad \operatorname{curl} \mathbf{H}=\mathbf{J}+\sigma \mathbf{E}, \\
& \operatorname{div}(\varepsilon \mathbf{E})=0, \quad \operatorname{div}(\mu \mathbf{H})=0,
\end{aligned}
$$

where $\mu, \sigma$ and $\varepsilon$ represent the physical (scalar) parameters respectively called magnetic permeability, electric conductivity and electric permittivity.

We assume that these parameters are piecewise smooth real valued functions satisfying:

$$
\begin{array}{lllll}
\varepsilon_{\max } \geq \varepsilon(\mathbf{x}) \geq \varepsilon_{\min }>0 & \text { a.e. in } \hat{\Omega}_{\mathrm{C}} \quad \text { and } & \varepsilon(\mathbf{x})=\varepsilon_{\min } & \text { a.e. in } \mathbb{R}^{3} \backslash \overline{\hat{\Omega}}_{\mathrm{C}} \\
\sigma_{\max } \geq \sigma(\mathbf{x}) \geq \sigma_{\min }>0 & \text { a.e. in } \hat{\Omega}_{\mathrm{C}} \quad \text { and } & \sigma(\mathbf{x})=0 & \text { a.e. in } \mathbb{R}^{3} \backslash \overline{\hat{\Omega}}_{\mathrm{C}} \\
\mu_{\max } \geq \mu(\mathbf{x}) \geq \mu_{\min }>0 & \text { a.e. in } \hat{\Omega}_{\mathrm{C}} & \text { and } & \mu(\mathbf{x})=\mu_{\min } & \text { a.e. in } \mathbb{R}^{3} \backslash \overline{\hat{\Omega}}_{\mathrm{C}}
\end{array}
$$


Different formulations for the eddy current model ( $[5,13,24])$ can be analyzed as a degenerate parabolic problem of Section 3 and the mathematical analysis of their numerical approximation by using finite element methods can be obtained with the theory performed in Sections 4 and 5 , however we only focus in the formulation studied in the first of that references. Other formulations for the eddy current problem on a moving domain can be found in $[3,4]$. These formulations lead to parabolic degenerate problems more general than problems studied in Section 3 because the operator $R$ in that case is time-dependent.

Zlamal [24] (see also [25]) has proposed a solution of a particular case of the eddy current Problem 5 by solving the following two-dimensional degenerate parabolic problem, for a given data source $J_{\mathrm{D}}: \mathbb{R}^{2} \times[0, T] \rightarrow \mathbb{R}$.

Problem 6. Find $u: \mathbb{R}^{2} \times[0, T] \rightarrow \mathbb{R}$ such that

$$
\sigma \frac{\partial u}{\partial t}=\operatorname{div}\left(\frac{1}{\mu} \nabla u\right)+J_{\mathrm{D}}
$$

where the physical parameters $\sigma$ and $\mu$ are independent of $x_{3}$.

The following result shows the relationship between the eddy current Problem 5 and the degenerate parabolic equation Problem 6.

Proposition 2. If $u: \mathbb{R}^{2} \times[0, T] \rightarrow \mathbb{R}$ is an enough regular solution of Problem 6 and the electric permittivity $\varepsilon$ is independent of $x_{3}$, then

$$
\boldsymbol{E}:=\left(0,0,-\frac{\partial u}{\partial t}\right) \quad \text { and } \quad \boldsymbol{H}:=\frac{1}{\mu}\left(\frac{\partial u}{\partial x_{2}},-\frac{\partial u}{\partial x_{1}}, 0\right)
$$

are solutions of problem Problem 5 with $\mathbf{J}:=\left(0,0, J_{\mathrm{D}}\right)$.

Proof. Let $u$ be a regular solution of Problem 6 and assume that $\mathbf{J}:=\left(0,0, J_{\mathrm{D}}\right)$. Let us define $\boldsymbol{E}$ and $\boldsymbol{H}$ as in (6.2). Therefore,

$$
\operatorname{curl} \boldsymbol{E}=\left(-\frac{\partial}{\partial x_{2}}\left(\frac{\partial u}{\partial t}\right), \frac{\partial}{\partial x_{1}}\left(\frac{\partial u}{\partial t}\right), 0\right)=-\frac{\partial}{\partial t}(\mu \boldsymbol{H}),
$$

and the first equation of Problem 5 follows. Furthermore, the second equation of Problem 6 is obtained by noticing that

$$
\begin{aligned}
\operatorname{curl} \boldsymbol{H} & =\left(0,0,-\frac{\partial}{\partial x_{1}}\left(\frac{1}{\mu} \frac{\partial u}{\partial x_{1}}\right)-\frac{\partial}{\partial x_{2}}\left(\frac{1}{\mu} \frac{\partial u}{\partial x_{2}}\right)\right) \\
& =\left(0,0,-\operatorname{div}\left(\frac{1}{\mu} \nabla u\right)\right)=\mathbf{J}+\sigma \boldsymbol{E} .
\end{aligned}
$$

Next, by recalling that $u$ and $\varepsilon$ are independent of $x_{3}$, it follows the third equation of Problem 5. Finally, the last equation of Problem 5 follows by using the regularity of $u$. 


\subsection{Well-posedness for the eddy current formulation}

Let $\hat{\Omega} \subset \mathbb{R}^{3}$ be an open, bounded and simply connected set containing $\hat{\Omega}_{\mathrm{C}}$ and Supp $\mathbf{J}$, with $\mathbf{J}$ as in Proposition 2. In order to obtain a weak formulation for Problem 6, we have to consider the projection of both sets $\hat{\Omega}$ and the conducting domain $\hat{\Omega}_{\mathrm{C}}$ onto the plane $x_{1} x_{2}$, that will be denoted respectively as $\Omega$ and $\Omega_{\mathrm{C}}$. We can notice that in this case $\bar{\Omega}_{\mathrm{C}} \subset \Omega$. Therefore, given $u_{\mathrm{C}}^{0} \in \mathrm{L}^{2}\left(\Omega_{\mathrm{C}}\right)$ and $J_{\mathrm{D}} \in \mathrm{L}^{2}\left(0, T ; \mathrm{L}^{2}(\Omega)\right)$, by multiplying equation (6.1) with $v \in \mathrm{H}_{0}^{1}(\Omega)$ and integrating by parts over $\Omega$, we obtain the following weak formulation for the Problem 6.

Problem 7. Find $u \in \mathrm{L}^{2}\left(0, T ; \mathrm{H}_{0}^{1}(\Omega)\right)$ such that

$$
\begin{aligned}
\frac{d}{d t} \int_{\Omega_{\mathrm{C}}} \sigma u v+\int_{\Omega} \frac{1}{\mu} \nabla u \cdot \nabla v & =\int_{\Omega} J_{\mathrm{D}} v, & & \forall v \in \mathrm{H}_{0}^{1}(\Omega), \\
\left.u(0)\right|_{\Omega_{\mathrm{C}}} & =u_{\mathrm{C}}^{0} & & \text { in } \Omega_{\mathrm{C}} .
\end{aligned}
$$

The analysis of existence and uniqueness of solution for the previous problem is obtained by using Theorem 1. For this purpose, in order to fit Problem 7 in the abstract structure of Problem 1, we have to define $X:=\mathrm{H}_{0}^{1}(\Omega)$ and $Y:=\mathrm{L}^{2}(\Omega)$, with their usual inner products. Furthermore, we define the operators $R: Y \rightarrow Y^{\prime}$ and $A: X \rightarrow X^{\prime}$ given by

$$
\begin{aligned}
\langle A v, w\rangle_{X}:=\int_{\Omega} \frac{1}{\mu} \nabla v \cdot \nabla w, & \forall v, w \in X, \\
\langle R v, w\rangle_{Y}:=\int_{\Omega_{\mathrm{C}}} \sigma v w, & \forall v, w \in Y .
\end{aligned}
$$

We can notice that in this case the family of operators $A(t)$ in Problem 1 is constant with respect of $t$ and the functional spaces and operators satisfy the corresponding properties of Section 3. Moreover, it is easy to check that

$$
\hat{R} v=\sigma \chi_{\Omega_{\mathrm{C}}} v, \quad \hat{R}^{1 / 2} v=\sigma^{1 / 2} \chi_{\Omega_{\mathrm{C}}} v, \quad \forall v \in Y:=\mathrm{L}^{2}(\Omega),
$$

where $\chi_{\Omega_{\mathrm{C}}}$ is the characteristic function of $\Omega_{\mathrm{C}}$. Furthermore,

$$
Y_{+}^{1 / 2}=Y_{+}=\left\{v \in \mathrm{L}^{2}(\Omega):\left.v\right|_{\Omega \backslash \bar{\Omega}_{\mathrm{C}}}=0\right\} \cong \mathrm{L}^{2}\left(\Omega_{\mathrm{C}}\right)
$$

and $P_{+} v=\chi_{\Omega_{\mathrm{C}}} v$ for all $v \in Y$.

Additionally, we need to define the function $f \in \mathrm{L}^{2}\left(0, T ; X^{\prime}\right)$ given by

$$
\langle f(t), v\rangle_{X}:=\int_{\Omega} J_{\mathrm{D}}(t) v, \quad \forall v \in X
$$

Finally, we should notice that the initial condition of Problem 7 is equivalent to $R u(0)=R u_{0}$ in $Y^{\prime}$, where $u_{0}:=\widetilde{u}_{\mathrm{C}}^{0} \in Y$ is the extension by zero of $u_{\mathrm{C}}^{0}$ to the whole $\Omega$.

Theorem 4. There exists a unique solution u of Problem 7 satisfying

$$
\|u\|_{\mathrm{L}^{2}\left(0, T ; \mathrm{H}_{0}^{1}(\Omega)\right)} \leq C\left\{\left\|u_{\mathrm{C}}^{0}\right\|_{\mathrm{L}^{2}\left(\Omega_{\mathrm{C}}\right)}+\left\|J_{\mathrm{D}}\right\|_{\mathrm{L}^{2}\left(0, T ; \mathrm{L}^{2}(\Omega)\right)}\right\} .
$$


Proof. The operator $R$ is clearly monotone and self-adjoint. Furthermore, the following Garding-type inequality holds true for all $v \in X$ :

$$
\begin{aligned}
\langle R v, v\rangle_{Y}+\langle A v, v\rangle_{X} & =\int_{\Omega_{\mathrm{C}}} \sigma|v|^{2}+\int_{\Omega} \frac{1}{\mu}|\nabla v|^{2} \\
& \geq \frac{1}{\mu_{\max }} \int_{\Omega}|\nabla v|^{2} \geq \frac{C_{\mathrm{P}}}{\mu_{\max }}\|v\|_{\mathrm{H}^{1}(\Omega)}^{2},
\end{aligned}
$$

where $C_{\mathrm{P}}$ is the positive constant given by the Poincaré inequality in $\mathrm{H}_{0}^{1}(\Omega)$. Consequently, Theorem 1 shows that Problem 7 has at least a solution. Moreover, since the family of operators $A$ is independent of time, it is trivially a regular family and consequently the solution $u$ of Problem 7 is unique. Finally, by using (3.2) and noticing that

$$
\left\langle R u_{0}, u_{0}\right\rangle_{Y}=\int_{\Omega_{\mathrm{C}}} \sigma\left|u_{0}\right|^{2} \leq \sigma_{\max }\left\|u_{\mathrm{C}}^{0}\right\|_{\mathrm{L}^{2}\left(\Omega_{\mathrm{C}}\right)}^{2},
$$

we conclude the proof.

Remark \%. It is easy to see that

$$
\sigma \partial_{t} u-\operatorname{div}\left(\frac{1}{\mu} \nabla u\right)=J_{\mathrm{D}} \quad \text { in } \mathrm{L}^{2}\left(0, T ; \mathrm{H}_{0}^{1}(\Omega)^{\prime}\right),
$$

consequently $\sigma \partial_{t} u \in L^{2}\left(0, T ; \mathrm{H}_{0}^{1}\left(\Omega_{\mathrm{C}}\right)^{\prime}\right)$ and it is given by

$$
\left\langle\sigma \partial_{t} u, \varphi\right\rangle=\int_{\Omega_{\mathrm{C}}} J_{\mathrm{D}} \varphi-\int_{\Omega_{\mathrm{C}}} \frac{1}{\mu} \nabla u \cdot \nabla \varphi, \quad \forall \varphi \in \mathrm{H}_{0}^{1}\left(\Omega_{\mathrm{C}}\right) .
$$

Therefore $\left.u\right|_{\Omega_{\mathrm{C}}}$ belongs to the space $W^{1,2}\left(0, T ; \mathrm{H}^{1}\left(\Omega_{\mathrm{C}}\right), \mathrm{H}_{0}^{1}\left(\Omega_{\mathrm{C}}\right)^{\prime}\right)$.

\subsection{Error estimates for the fully-discrete degenerate formulation}

The fully-discrete approximation for the degenerate Problem 7 is obtained by using a finite element subspaces to define $X_{h}$ which is the corresponding family of finite dimensional subspaces of $X$ (see Section 4). For this purpose, in what follows we assume that $\Omega$ and $\Omega_{\mathrm{C}}$ are Lipschitz polygonal. Let $\left\{\mathcal{T}_{h}\right\}_{h}$ be a regular family of triangles meshes of $\Omega$ such that each element $K \in \mathcal{T}_{h}$ is contained either in $\bar{\Omega}_{\mathrm{c}}$ or in $\bar{\Omega}_{\mathrm{d}}:=\overline{\Omega \backslash \bar{\Omega}_{\mathrm{C}}}$. As usual, $h$ stands for the largest diameter of the triangles $K$ in $\mathcal{T}_{h}$.

We define $X_{h}$ using the standard Lagrange finite element subspace of $\mathrm{H}_{0}^{1}(\Omega)$, i.e.,

$$
X_{h}:=\left\{v_{h} \in C^{0}(\bar{\Omega}):\left.v\right|_{K} \in \mathbb{P}_{1}(K)\right\} \cap \mathrm{H}_{0}^{1}(\Omega),
$$

where $C^{0}(\bar{\Omega})$ is the space of scalar continuous functions defined on $\bar{\Omega}$ and $\mathbb{P}_{1}$ is the set of polynomials of degree not greater than 1 . Then, the fully-discrete approximation for the degenerate parabolic formulation is given by Problem 4, by using the notation (6.3)-(6.5). More precisely if $J_{\mathrm{D}} \in \mathcal{C}^{0}\left([0, T] ; \mathrm{L}^{2}(\Omega)\right)$ and $u_{0, h} \in X_{h}$, the fully-discrete approximation of Problem 7 can be read as follows. 
Problem 8. Find $u_{h}^{n} \in X_{h}, n=1, \ldots, N$, such that

$$
\begin{aligned}
\int_{\Omega_{\mathrm{C}}} \sigma\left(\frac{u_{h}^{n}-u_{h}^{n-1}}{\Delta t}\right) v+\int_{\Omega} \frac{1}{\mu} \nabla u_{h}^{n} \cdot \nabla v & =\int_{\Omega} J_{\mathrm{D}}\left(t_{n}\right) v, \quad \forall v \in X_{h}, \\
R u_{h}^{0} & =R u_{0, h} .
\end{aligned}
$$

Thus, by using (6.6), the existence and uniqueness of solution $u_{h}^{n} \in X_{h}$, $n=1, \ldots, N$, of the fully-discrete problem is guaranteed by Theorem 2 for a small enough time-step.

In order to to obtain a suitable error estimate for the approximation and according to the analysis at the end of Section 5 (cf. Remark 6), we can take advantage of the "allowed freedom" to choice both the initial data $u_{0}$ to analyze Problem 7 and the discrete initial data $u_{0, h}$ in Problem 8 (see [4] for similar ideas). To do this, in what follows we assume that $u_{\mathrm{C}}^{0} \in \mathrm{H}^{1}\left(\Omega_{\mathrm{C}}\right)$ (cf. Problem 7$)$.

Let $u_{\mathrm{D}}^{0} \in \mathrm{H}^{1}\left(\Omega_{\mathrm{D}}\right)$ such that $\left.u_{\mathrm{D}}^{0}\right|_{\partial \Omega_{\mathrm{C}}}=\left.u_{\mathrm{C}}^{0}\right|_{\partial \Omega_{\mathrm{C}}},\left.u_{\mathrm{D}}^{0}\right|_{\partial \Omega}=0$ and satisfying

$$
\int_{\Omega_{\mathrm{D}}} \frac{1}{\mu} \nabla u_{\mathrm{D}}^{0} \cdot \nabla v=\int_{\Omega_{\mathrm{D}}} J_{\mathrm{D}}(0) v, \quad \forall v \in \mathrm{H}_{0}^{1}\left(\Omega_{\mathrm{D}}\right) .
$$

Consequently, if we define

$$
u_{0}:= \begin{cases}u_{\mathrm{C}}^{0} & \text { in } \Omega_{\mathrm{C}} \\ u_{\mathrm{D}}^{0} & \text { in } \Omega_{\mathrm{D}}\end{cases}
$$

then $u_{0} \in \mathrm{H}_{0}^{1}(\Omega)$ and the initial condition in Problem 7 is equivalent to $R u(0)=$ $R u_{0}$. Furthermore, we can notice that

$$
X_{0}:=\mathrm{H}_{0}^{1}(\Omega) \cap \operatorname{ker} R=\left\{\widetilde{v}: v \in \mathrm{H}_{0}^{1}\left(\Omega_{\mathrm{D}}\right)\right\},
$$

where $\widetilde{v}$ is the extension by zero of $v$ to the whole $\Omega$, hence from (5.30) and (6.7) it follows that $u_{0}=\mathcal{E} u_{0}$.

We now proceed to define $u_{0, h}$. To this end, we introduce the following notation

$$
X_{h}\left(\Omega_{\mathrm{C}}\right):=\left\{\left.v_{h}\right|_{\Omega_{\mathrm{C}}}: v_{h} \in X_{h}\right\}, \quad X_{h}\left(\Omega_{\mathrm{D}}\right):=\left\{\left.v_{h}\right|_{\Omega_{\mathrm{D}}}: v_{h} \in X_{h}\right\}
$$

and $X_{h}^{0}\left(\Omega_{\mathrm{D}}\right):=X_{h}\left(\Omega_{\mathrm{D}}\right) \cap \mathrm{H}_{0}^{1}\left(\Omega_{\mathrm{D}}\right)$.

Let $u_{\mathrm{C}, h}^{0}$ be an arbitrary function in $X_{h}\left(\Omega_{\mathrm{C}}\right)$ and $u_{\mathrm{D}, h}^{0} \in X_{h}\left(\Omega_{\mathrm{D}}\right)$ such that $\left.u_{\mathrm{D}, h}^{0}\right|_{\partial \Omega_{\mathrm{C}}}=\left.u_{\mathrm{C}, h}^{0}\right|_{\partial \Omega_{\mathrm{C}}},\left.u_{\mathrm{D}, h}^{0}\right|_{\partial \Omega}=0$ and satisfying

$$
\int_{\Omega_{\mathrm{D}}} \frac{1}{\mu} \nabla u_{\mathrm{D}, h}^{0} \cdot \nabla v_{h}=\int_{\Omega_{\mathrm{D}}} J_{\mathrm{D}}(0) v_{h} \quad \forall v_{h} \in X_{h}^{0}\left(\Omega_{\mathrm{D}}\right) .
$$

We can notice that $u_{\mathrm{D}, h}^{0}$ is the discrete finite element approximation for the solution of Problem (6.7), hence by proceeding analogously to [17, Section 6.2.1], we deduce

$$
\left\|u_{\mathrm{D}}^{0}-u_{\mathrm{D}, h}^{0}\right\|_{\mathrm{H}^{1}\left(\Omega_{\mathrm{D}}\right)} \leq C \inf _{w_{h} \in X_{h}^{*}\left(\Omega_{\mathrm{D}}\right)}\left\|u_{\mathrm{D}}^{0}-w_{h}\right\|_{\mathrm{H}^{1}\left(\Omega_{\mathrm{D}}\right)}
$$


where

$$
X_{h}^{*}\left(\Omega_{\mathrm{D}}\right):=\left\{w_{h} \in X_{h}\left(\Omega_{\mathrm{D}}\right):\left.w_{h}\right|_{\partial \Omega_{\mathrm{C}}}=\left.u_{\mathrm{C}, h}^{0}\right|_{\partial \Omega_{\mathrm{C}}}\right\}
$$

Therefore, by defining

$$
u_{0, h}:= \begin{cases}u_{\mathrm{C}, h}^{0} & \text { in } \Omega_{\mathrm{C}} \\ u_{\mathrm{D}, h}^{0} & \text { in } \Omega_{\mathrm{D}}\end{cases}
$$

then $u_{0, h} \in X_{h}$ and it can be considered as the initial data in Problem 8 . Moreover, by recalling (5.32) it follows immediately that $\mathcal{E}_{h} u_{0, h}=u_{0, h}$. Consequently if the solution of Problem 7 satisfies $u \in \mathcal{C}^{0}\left([0, T] ; \mathrm{H}_{0}^{1}(\Omega)\right), u_{0}$ and $u_{0, h}$ are given by (6.8) and (6.10) respectively, the analysis in Remark 6 (see $(5.35))$ shows that

$$
u(0)=u_{0} .
$$

Then, from (6.9) it follows that there exists a constant $C>0$ such that

$$
\begin{aligned}
\left\|u(0)-u_{0, h}\right\|_{\mathrm{H}_{0}^{1}(\Omega)} & =\left\|u_{0}-u_{0, h}\right\|_{\mathrm{H}_{0}^{1}(\Omega)} \\
& \leq C\left\{\left\|u_{\mathrm{C}}^{0}-u_{\mathrm{C}, h}^{0}\right\|_{\mathrm{H}^{1}\left(\Omega_{\mathrm{C}}\right)}+\inf _{w \in X_{h}^{*}\left(\Omega_{\mathrm{D}}\right)}\left\|u_{\mathrm{D}}^{0}-w\right\|_{\mathrm{H}^{1}\left(\Omega_{\mathrm{D}}\right)}\right\} .
\end{aligned}
$$

Finally, by noticing that in this case we have

$$
\left\|\partial_{t}\left(P_{+} u\right)\left(t_{k}\right)-\bar{\partial} P_{+} u_{h}^{k}\right\|_{+}^{2}=\int_{\Omega_{\mathrm{C}}} \sigma\left|\partial_{t} u\left(t_{k}\right)-\bar{\partial} u_{h}^{k}\right|^{2}
$$

we obtain the following result about the error estimates for the fully-discrete approximation of the degenerate parabolic Problem 7 (cf. Problem 8), which is a direct consequence of Theorem 3 .

Theorem 5. Let $u_{\mathrm{C}}^{0} \in \mathrm{H}^{1}\left(\Omega_{\mathrm{C}}\right)$ and $u_{\mathrm{C}, h}^{0} \in X_{h}\left(\Omega_{\mathrm{C}}\right), u_{0} \in \mathrm{H}_{0}^{1}(\Omega)$ and $u_{0, h} \in X_{h}$ given by (6.8) and (6.10) respectively. Let $u$ be the solution of the eddy current Problem 7 and $u_{h}^{n} \in X_{h}(n=1, \ldots, N)$ the fully-discrete solution of Problem 8.

If $u \in \mathrm{H}^{1}\left(0, T ; \mathrm{H}_{0}^{1}(\Omega)\right)$ with $\left.u\right|_{\Omega_{\mathrm{C}}} \in \mathrm{H}^{2}\left(0, T ; \mathrm{L}^{2}\left(\Omega_{\mathrm{C}}\right)\right)$ then there exists a constant $C>0$, independent of $h$ and $\Delta t$, such that

$$
\begin{aligned}
& \max _{1 \leq n \leq N}\left\|u\left(t_{n}\right)-u_{h}^{n}\right\|_{\sigma}^{2}+\Delta t \sum_{n=1}^{N}\left\|u\left(t_{n}\right)-u_{h}^{n}\right\|_{\mathrm{H}_{0}^{1}(\Omega)}^{2}+\Delta t \sum_{n=1}^{N}\left\|\partial_{t} u\left(t_{n}\right)-\bar{\partial} u_{h}^{n}\right\|_{\sigma}^{2} \\
& \leq C\left\{\left\|u_{\mathrm{C}}^{0}-u_{\mathrm{C}, h}^{0}\right\|_{\mathrm{H}^{1}\left(\Omega_{\mathrm{C}}\right)}^{2}+\inf _{w \in X_{h}^{*}\left(\Omega_{\mathrm{D}}\right)}\left\|u_{\mathrm{D}}^{0}-w\right\|_{\mathrm{H}^{1}\left(\Omega_{\mathrm{D}}\right)}^{2}\right. \\
& +\max _{0 \leq n \leq N}\left[\inf _{v \in X_{h}}\left\|u\left(t_{n}\right)-v\right\|_{\mathrm{H}_{0}^{1}(\Omega)}^{2}\right]+\int_{0}^{T} \inf _{v \in X_{h}}\left\|\partial_{t} u(t)-v\right\|_{\mathrm{H}_{0}^{1}(\Omega)}^{2} d t \\
& \left.+(\Delta t)^{2} \int_{0}^{T}\left\|\partial_{t t} u(t) d t\right\|_{\mathrm{L}^{2}\left(\Omega_{\mathrm{C}}\right)}^{2}\right\}, \text { where }\|w\|_{\sigma}^{2}:=\int_{\Omega_{\mathrm{C}}} \sigma|w|^{2} .
\end{aligned}
$$

Finally, to obtain the asymptotic error estimate, we need to consider the Sobolev space $\mathrm{H}^{1+s}(\Omega)$ for $0<s \leq 1$. It is well known that the Lagrange 
interpolant $\mathcal{L}_{h} v \in X_{h}$ is well defined for all $v \in \mathrm{H}_{0}^{1}(\Omega) \cap \mathrm{H}^{1+s}(\Omega)$ and satisfies the following estimate (see, for instance, [8])

$$
\left\|v-\mathcal{L}_{h} v\right\|_{\mathrm{H}^{1}(\Omega)} \leq C h^{s}\|v\|_{\mathrm{H}^{1+s}(\Omega)}, \quad \forall v \in \mathrm{H}_{0}^{1}(\Omega) \cap \mathrm{H}^{1+s}(\Omega) .
$$

Consequently, we have the following result which shows the asymptotic convergence of the fully-discrete approximation.

Corollary 1. Let $u_{\mathrm{C}}^{0} \in \mathrm{H}^{1}\left(\Omega_{\mathrm{C}}\right)$ and let $u_{0} \in \mathrm{H}_{0}^{1}(\Omega)$ given by (6.8). Assume that

$$
u \in \mathrm{H}^{1}\left(0, T ; \mathrm{H}_{0}^{1}(\Omega) \cap \mathrm{H}^{1+s}(\Omega)\right) \text { with }\left.u\right|_{\Omega_{\mathrm{C}}} \in \mathrm{H}^{2}\left(0, T ; \mathrm{L}^{2}\left(\Omega_{\mathrm{C}}\right)\right) .
$$

Then, if $u_{\mathrm{C}, h}^{0}:=\left.\left(\mathcal{L}_{h} u_{0}\right)\right|_{\Omega_{\mathrm{C}}}$ and $u_{0, h}$ is defined by $(6.10)$, there exists a constant $C>0$ independent of $h$ and $\Delta t$, such that

$$
\begin{aligned}
& \max _{1 \leq n \leq N}\left\|u\left(t_{n}\right)-u_{h}^{n}\right\|_{\sigma}^{2}+\Delta t \sum_{n=1}^{N}\left\|u\left(t_{n}\right)-u_{h}^{n}\right\|_{\mathrm{H}_{0}^{1}(\Omega)}^{2}+\Delta t \sum_{n=1}^{N}\left\|\partial_{t} u\left(t_{n}\right)-\bar{\partial} u_{h}^{n}\right\|_{\sigma}^{2} \\
& \leq C\left\{h^{2 s}\left[\left\|u_{0}\right\|_{\mathrm{H}^{1+s}(\Omega)}^{2}+\max _{1 \leq n \leq N}\left\|u\left(t_{n}\right)\right\|_{\mathrm{H}^{1+s}(\Omega)}^{2}+\left\|\partial_{t} u\right\|_{\mathrm{L}^{2}\left(0, T ; \mathrm{H}^{1+s}(\Omega)\right)}\right]\right. \\
& \left.\quad+(\Delta t)^{2}\left\|\partial_{t t} u\right\|_{\mathrm{L}^{2}\left(0, T ; \mathrm{L}^{2}\left(\Omega_{\mathrm{C}}\right)\right)}^{2}\right\}
\end{aligned}
$$

Proof. We firstly notice that since $u(0)=u_{0}$ (see $(6.11)$ ), the regularity of $u$ implies $u_{0} \in \mathrm{H}_{0}^{1}(\Omega) \cap \mathrm{H}^{1+s}(\Omega)$ and $\mathcal{L}_{h} u_{0} \in X_{h}$ is well defined. Hence $u_{\mathrm{C}, h}^{0}:=\left.\left(\mathcal{L}_{h} u_{0}\right)\right|_{\Omega_{\mathrm{C}}} \in X_{h}\left(\Omega_{\mathrm{C}}\right)$ is also well defined and

$$
\left.\left\|u_{\mathrm{C}}^{0}-u_{\mathrm{C}, h}^{0}\right\|_{\mathrm{H}^{1}\left(\Omega_{\mathrm{C}}\right)} \leq \| u_{0}-\mathcal{L}_{h} u_{0}\right) \|_{\mathrm{H}^{1}(\Omega)} .
$$

On the other hand, if $u_{\mathrm{D}, h}^{*}:=\left.\left(\mathcal{L}_{h} u_{0}\right)\right|_{\Omega_{\mathrm{D}}}$ then $u_{\mathrm{D}, h}^{*} \in X_{h}^{*}\left(\Omega_{\mathrm{D}}\right)$ and

$$
\inf _{w \in X_{h}^{*}\left(\Omega_{\mathrm{D}}\right)}\left\|u_{\mathrm{D}}^{0}-w\right\|_{\mathrm{H}^{1}\left(\Omega_{\mathrm{D}}\right)} \leq\left\|u_{\mathrm{D}}^{0}-u_{\mathrm{D}, h}^{*}\right\|_{\mathrm{H}^{1}\left(\Omega_{\mathrm{D}}\right)} \leq\left\|u_{0}-\mathcal{L}_{h} u_{0}\right\|_{\mathrm{H}^{1}(\Omega)} .
$$

Consequently, the result follows from Theorem 5 and the interpolation error estimate (6.12).

Remark 8. If the discrete initial condition $u_{\mathrm{C}, h}^{0}$ is chosen as in Corollary 1 (i.e. $\left.u_{\mathrm{C}, h}^{0}:=\left.\left(\mathcal{L}_{h} u_{0}\right)\right|_{\Omega_{\mathrm{C}}}\right)$ the obtention of the error estimate can be further simplified. There is no need to introduce $u_{\mathrm{D}, h}^{0}$. It is enough to take (in lieu of (6.10)) $u_{0, h}:=\mathcal{L}_{h} u_{0} \in X_{h}$, what is allowed due to $\left.\left(\mathcal{L}_{h} u_{0}\right)\right|_{\Omega_{\mathrm{C}}}=u_{\mathrm{C}, h}^{0}$ and the definition of operator $R$ (see (6.4)). Since $u_{0}$ is defined by $(6.8)$, we have $u(0)=u_{0}$ and the term $\left\|u(0)-u_{0, h}\right\|_{X}$ in $(5.27)$ reduces simply to $\left\|u_{0}-u_{0, h}\right\|_{\mathrm{H}^{1}(\Omega)}=$ $\left\|u_{0}-\mathcal{L}_{h} u_{0}\right\|_{\mathrm{H}^{1}(\Omega)}$.

The technique based on the introduction of $u_{\mathrm{D}, h}^{0}$ and estimate (6.9) has a more general scope since it allows to consider an arbitrary discrete initial condition $u_{\mathrm{C}, h}^{0} \in X_{h}\left(\Omega_{\mathrm{C}}\right)$ such that $\lim _{h \rightarrow 0^{+}}\left\|u_{\mathrm{C}}^{0}-u_{\mathrm{C}, h}^{0}\right\|_{\mathrm{H}^{1}\left(\Omega_{\mathrm{C}}\right)}=0$ and not necessarily $u_{\mathrm{C}, h}^{0}=\left.\left(\mathcal{L}_{h} u_{0}\right)\right|_{\Omega_{\mathrm{C}}}$. In fact, since the family of triangle meshes of $\Omega$ is regular, if we define $X_{h}\left(\partial \Omega_{\mathrm{C}}\right):=\left\{\left.z_{h}\right|_{\partial \Omega_{\mathrm{C}}}: z_{h} \in X_{h}\right\}$, there exists a discrete 
lifting operator (see $\left[6\right.$, Theorem 5.1]) $R_{h}: X_{h}\left(\partial \Omega_{\mathrm{C}}\right) \rightarrow X_{h}\left(\Omega_{\mathrm{D}}\right)$ and a constant $C>0$ independent of $h$ such that for $h$ small enough the inequality

$$
\left\|R_{h} g\right\|_{\mathrm{H}^{1}\left(\Omega_{\mathrm{D}}\right)} \leq C\|g\|_{\mathrm{H}^{1 / 2}\left(\partial \Omega_{\mathrm{C}}\right)}, \quad \forall g \in X_{h}\left(\partial \Omega_{\mathrm{C}}\right)
$$

holds. Using this, it is easy to obtain that

$$
\begin{aligned}
& \inf _{w \in X_{h}^{*}\left(\Omega_{\mathrm{D}}\right)}\left\|u_{\mathrm{D}}^{0}-w\right\|_{\mathrm{H}^{1}\left(\Omega_{\mathrm{D}}\right)} \\
& \leq C\left\{\left\|u_{\mathrm{C}}^{0}-u_{\mathrm{C}, h}^{0}\right\|_{\mathrm{H}^{1}\left(\Omega_{\mathrm{C}}\right)}+\inf _{w \in X_{h}\left(\Omega_{\mathrm{D}}\right)}\left\|u_{\mathrm{D}}^{0}-w\right\|_{\mathrm{H}^{1}\left(\Omega_{\mathrm{D}}\right)}\right\} .
\end{aligned}
$$

The convergence (and the corresponding error estimate under the assumption $\left.\left\|u_{\mathrm{C}}^{0}-u_{\mathrm{C}, h}^{0}\right\|_{\mathrm{H}^{1}\left(\Omega_{\mathrm{C}}\right)}=O\left(h^{s}\right)\right)$ follows from Theorem 5 by using an interpolation error estimate like (6.12) to bound the infimum in the right hand side of (6.13).

Remark 9. Corollary 1 shows that the fully-discrete approximation Problem 8 provides a suitable approximation for the physical variables of the eddy current problem at each time $t_{n}$, namely the electric field $\mathbf{E}\left(t_{n}\right)$ in the three-dimensional conducting domain $\hat{\Omega}_{\mathrm{C}}$ and the magnetic field $\mathbf{H}\left(t_{n}\right)$ in the three-dimensional computational domain $\hat{\Omega}$. More precisely, we can use the relationship (6.2), to define

$$
\mathbf{E}\left(t_{n}\right):=\left(0,0,-\partial_{t} u\left(t_{n}\right)\right) \text { in } \hat{\Omega}_{\mathrm{C}}, \quad \mathbf{H}\left(t_{n}\right):=\frac{1}{\mu}\left(\frac{\partial u}{\partial x_{2}}\left(t_{n}\right),-\frac{\partial u}{\partial x_{1}}\left(t_{n}\right), 0\right) \text { in } \hat{\Omega},
$$

for any $n=1, \ldots, N$, and propose the following approximations

$$
\begin{aligned}
& \mathbf{E}\left(t_{n}\right) \approx \mathbf{E}_{h}^{n}:=\left(0,0,-\bar{\partial} u_{h}^{n}\right) \quad \text { in } \hat{\Omega}_{\mathrm{C}}, \\
& \mathbf{H}\left(t_{n}\right) \approx \mathbf{H}_{h}^{n}:=\frac{1}{\mu}\left(\frac{\partial u_{h}^{n}}{\partial x_{2}},-\frac{\partial u_{h}^{n}}{\partial x_{1}}, 0\right) \quad \text { in } \hat{\Omega} .
\end{aligned}
$$

Consequently, by using Corollary 1 , we deduce the following quasi-optimal error estimates

$$
\Delta t \sum_{n=1}^{N}\left\|\mathbf{E}\left(t_{n}\right)-\mathbf{E}_{h}^{n}\right\|_{\sigma}^{2}+\Delta t \sum_{n=1}^{N}\left\|\mathbf{H}\left(t_{n}\right)-\mathbf{H}_{h}^{n}\right\|_{\mu, \hat{\Omega}}^{2} \leq C\left[h^{2 s}+(\Delta t)^{2}\right],
$$

where $\|\mathbf{w}\|_{\sigma}^{2}:=\int_{\hat{\Omega}_{\mathrm{C}}} \sigma|\mathbf{w}|^{2}$ and $\|\mathbf{w}\|_{\mu, \hat{\Omega}}^{2}:=\int_{\hat{\Omega}} \frac{1}{\mu}|\mathbf{w}|^{2}$.

\subsection{Numerical results}

In this subsection we present some numerical results obtained with a MATLAB code which implements the numerical method described in Problem 8, to illustrate the convergence with respect to the discretization parameters. To this end, we describe the results obtained for a test problem with a known analytical solution.

We consider $\hat{\Omega}$ with $\hat{\Omega}_{\mathrm{C}}$ and their respective projection onto the plane $x_{1} x_{2}$, $\Omega:=(0,1)^{2} \backslash[0.5,1]^{2}$ and $\Omega_{\mathrm{C}}:=(0.2,0.8)^{2} \backslash[0.4,0.8]^{2}$ (see Figure 1) and $T=1$. The right hand side $J_{\mathrm{D}}$, is chosen so that

$$
u\left(x_{1}, x_{2}, t\right)=e^{-5 \pi t} \sin \left(\pi x_{1}\right) \sin \left(\pi x_{2}\right),
$$



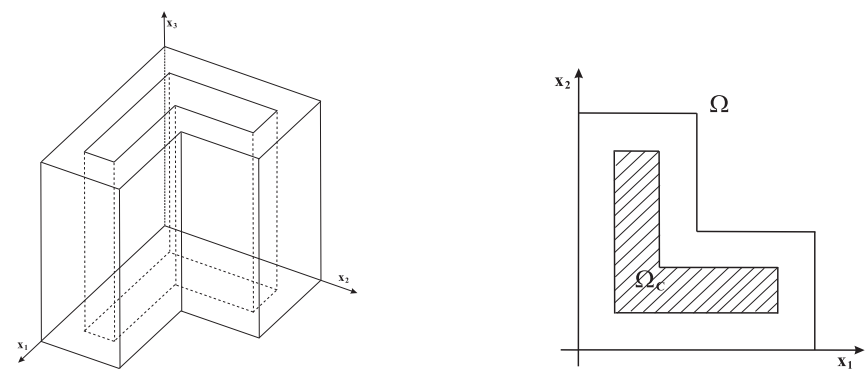

Figure 1. Sketch of the domain $3 \mathrm{D}$ (left) and 2D (right).

Table 1. Percentage errors for $\mathbf{H}$ in the $\mathrm{L}^{2}\left(0, T ; \mathrm{L}^{2}(\hat{\Omega})\right)$-norm, with $h=0.3687$ and $\Delta t=0.025$.

\begin{tabular}{lccccccc}
\hline \multicolumn{1}{c}{$h$} & $h / 2$ & $h / 4$ & $h / 8$ & $h / 16$ & $h / 32$ & $h / 64$ \\
\hline \multicolumn{1}{c}{$\Delta t$} & 41.369 & 22.1296 & 12.8925 & 9.1603 & 7.9516 & 7.6190 & 7.5335 \\
$\Delta t / 2$ & 41.309 & 21.4624 & 11.4341 & 6.8342 & 5.0574 & 4.5040 & 4.3546 \\
$\Delta t / 4$ & 41.445 & 21.3041 & 10.9212 & 5.8293 & 3.5396 & 2.6751 & 2.4108 \\
$\Delta t / 8$ & 41.582 & 21.3044 & 10.7883 & 5.5072 & 2.9460 & 1.845 & 1.3784 \\
$\Delta t / 16$ & 41.672 & 21.3307 & 10.7652 & 5.4225 & 2.7648 & 1.4813 & 0.9115 \\
$\Delta t / 32$ & 41.724 & 21.3514 & 10.7663 & 5.4038 & 2.7172 & 1.3851 & 0.7428 \\
$\Delta t / 64$ & 41.751 & 21.3637 & 10.7702 & 5.4008 & 2.7059 & 1.3599 & 0.6932 \\
\hline
\end{tabular}

is the solution to Problem 6 in $\Omega$ with boundary condition $u=0$ on $\partial \Omega$. Notice that $u$ is also solution of Problem 7 with $u_{0}\left(x_{1}, x_{2}\right)=\sin \left(\pi x_{1}\right) \sin \left(\pi x_{2}\right)$ where, in particular $u_{0} \in \mathrm{H}_{0}^{1}(\Omega) \cap \mathrm{H}^{2}(\Omega)$. We have taken $\mu=\mu_{0}=4 \pi \times 10^{-7} \mathrm{Hm}^{-1}$, $\sigma=\sigma=10^{6}(\Omega \mathrm{m})^{-1}$ in $\Omega_{\mathrm{C}}$, the magnetic permeability and electric conductivity of vacuum, respectively.

The numerical method has been applied with several successively refined meshes and time-steps. The computed approximate solutions have been compared with the analytical one, by calculating the relative percentage error in time-discrete norms from Corollary 1. More accurately, thanks to Proposition 2 and Remark 9, we have compute the relative percentage error for the physical variables of interest, the magnetic field and the electric field in the conductor domain, namely

$$
100 \frac{\Delta t \sum_{n=1}^{N}\left\|\mathbf{H}\left(t_{n}\right)-\mathbf{H}_{h}^{n}\right\|_{\mu, \hat{\Omega}}^{2}}{\Delta t \sum_{n=1}^{N}\left\|\mathbf{H}\left(t_{n}\right)\right\|_{\mu, \hat{\Omega}}^{2}}, \quad 100 \frac{\Delta t \sum_{n=1}^{N}\left\|\mathbf{E}\left(t_{n}\right)-\mathbf{E}_{h}^{n}\right\|_{\sigma, \hat{\Omega}_{\mathrm{C}}}^{2}}{\Delta t \sum_{n=1}^{N}\left\|\mathbf{E}\left(t_{n}\right)\right\|_{\sigma, \hat{\Omega}_{\mathrm{C}}}^{2}},
$$

which are time-discrete forms of the square of the errors in $\mathrm{L}^{2}\left(0, T ; \mathrm{L}^{2}(\hat{\Omega})\right)$ and $\mathrm{L}^{2}\left(0, T ; \mathrm{L}^{2}\left(\hat{\Omega}_{\mathrm{C}}\right)\right)$ norms, respectively.

The Table 1 shows the relative errors for $\mathbf{H}$ in the $\mathrm{L}^{2}\left(0, T ; \mathrm{L}^{2}(\hat{\Omega})\right)$-norm, 
Table 2. Percentage errors for $\mathbf{E}$ in the $\mathrm{L}^{2}\left(0, T ; \mathrm{L}^{2}\left(\Omega_{\mathrm{C}}\right)\right)$-norm, with $h=0.3687$ and $\Delta t=0.025$.

\begin{tabular}{lccccc}
\hline \multicolumn{1}{c}{$h$} & $h / 2$ & $h / 4$ & $h / 8$ & $h / 16$ \\
\hline \multicolumn{1}{c}{$\Delta t$} & 26.3489 & 23.9703 & 23.6728 & 23.6232 & 23.6127 \\
$\Delta t / 2$ & 17.2551 & 13.4472 & 13.1275 & 13.1028 & 13.1006 \\
$\Delta t / 4$ & 13.7947 & 7.5263 & 6.9433 & 6.9188 & 6.9213 \\
$\Delta t / 8$ & 13.2102 & 4.8159 & 3.6233 & 3.5566 & 3.5592 \\
$\Delta t / 16$ & 13.3954 & 3.9628 & 1.9873 & 1.8078 & 1.8042 \\
$\Delta t / 32$ & 13.6309 & 3.8427 & 1.3093 & 0.9290 & 0.9082 \\
$\Delta t / 64$ & 13.7873 & 3.8923 & 1.1142 & 0.5144 & 0.4574 \\
$\Delta t / 128$ & 13.8756 & 3.9494 & 1.0886 & 0.3501 & 0.2352 \\
$\Delta t / 256$ & 13.9223 & 3.9870 & 1.0992 & 0.3049 & 0.1323 \\
$\Delta t / 512$ & 13.9463 & 4.0081 & 1.1111 & 0.2992 & 0.0927
\end{tabular}
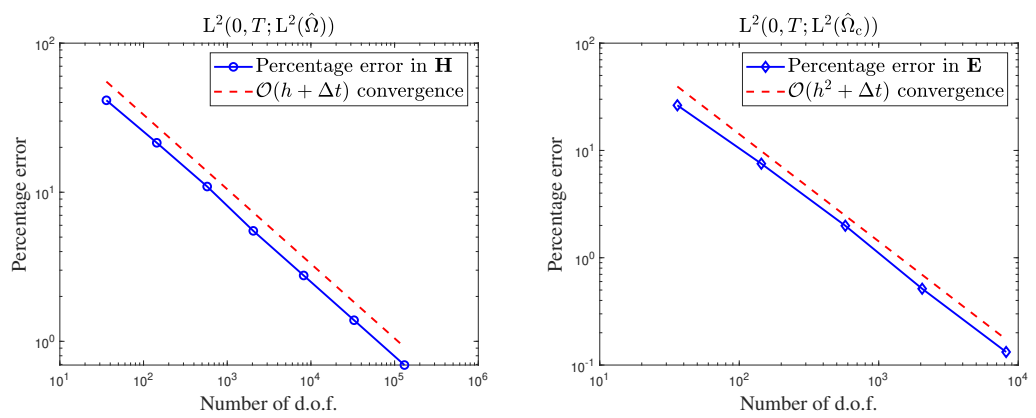

Figure 2. Percentage discretization error curves for $\mathbf{H}$ (left) and $\mathbf{E}$ (right) versus number of d.o.f. (log-log scale).

namely the relative errors for $u$ in the $\mathrm{L}^{2}\left(0, T ; \mathrm{H}_{0}^{1}(\Omega)\right)$-norm. We notice that by taking a small enough time-step $\Delta t$, we can observe the behavior of the error with respect to the space discretization (see the row corresponding to $\Delta t / 64)$. On the other hand, by considering a small enough mesh-size $h$, we can check the order convergence with respect $\Delta t$ (see the first entries of the column corresponding to $h / 64)$. Hence, we conclude an order the convergence $\mathcal{O}(h+\Delta t)$ for $\mathbf{H}$, which confirm the theoretical results given in Remark 9, proved in Corollary 1.

The Table 2 shows the relative errors for $\mathbf{E}$ in $\mathrm{L}^{2}\left(0, T ; \mathrm{L}^{2}\left(\hat{\Omega}_{\mathrm{C}}\right)\right)$, namely the relative errors $\partial_{t} u$ in the $\mathrm{L}^{2}\left(0, T ; \mathrm{L}^{2}\left(\Omega_{\mathrm{C}}\right)\right)$-norm. We proceed as above, now we can see an order the convergence $\mathcal{O}\left(h^{2}+\Delta t\right.$ ) (see the row corresponding to $\Delta t / 512$ and the column corresponding to $h / 16)$, in spite of the fact that only a linear order of convergence in $h$ has been proved above. Hence, we have obtained the theoretical results proved in Corollary 1, too.

Figure 2 shows log-log plots of the error of $\mathbf{H}$ (left) and $\mathbf{E}$ (right) versus number of degrees of freedom (d.o.f). To report this we have been values of $\Delta t$ 
proportional to $h$ (see the values within boxes in Table 1 ) and $\Delta t$ proportional to $h^{2}$ (see the values within boxes in Table 2), respectively. The slopes of the curves clearly show an order of convergence $\mathcal{O}(h+\Delta t)$ and $\mathcal{O}\left(h^{2}+\Delta t\right)$, respectively.

\section{Acknowledgements}

This work was partially supported by Colciencias through the 727 call, University of Cauca project VRI ID 5243 and by Universidad Nacional de Colombia through Hermes project 46332.

\section{References}

[1] A. Alonso-Rodríguez and A. Valli. Eddy current approximation of Maxwell equations. Theory, algorithms and applications, volume 4 of MSEA. Modeling, Simulation and Applications. Springer-Verlag Italia, Milan, 2010. https://doi.org/10.1007/978-88-470-1506-7.

[2] H. Ammari, A. Buffa and J.-C. Nédélec. A justification of eddy currents model for the Maxwell equations. SIAM J. Appl. Math., 60(5):1805-1823, 2000. https://doi.org/10.1137/S0036139998348979.

[3] A. Bermúdez, R. Mu noz Sola, C. Reales, R. Rodríguez and P. Salgado. A transient eddy current problem on a moving domain. Mathematical analysis. SIAM J. Math. Anal., 45(6):3629-3650, 2013. https://doi.org/10.1137/130914425.

[4] A.Bermúdez, R.Mu noz Sola, C.Reales, R.Rodríguez and P.Salgado. A transient eddy current problem on a moving domain. Numerical analysis. Adv. Comput. Math., 42(4):757-789, 2016. https://doi.org/10.1007/s10444-015-9441-0.

[5] A. Bermúdez, C. Reales, R. Rodríguez and P. Salgado. Numerical analysis of a transient eddy current axisymmetric problem involving velocity terms. Numer. Methods Partial Differential Equations, 28(3):984-1012, 2012. https://doi.org/10.1002/num.20670.

[6] C. Bernardi and V. Girault. A local regularization operator for triangular and quadrilateral finite elements. SIAM J. Numer. Anal, 35(5):1893-1916, 1998. https://doi.org/10.1137/S0036142995293766.

[7] A. Bossavit. Computational electromagnetism. Electromagnetism. Academic Press, Inc., San Diego, CA, 1998. Variational formulations, Complementarity, Edge elements.

[8] P.G. Ciarlet. The finite element method for elliptic problems, volume 40 of Classics in Applied Mathematics. Society for Industrial and Applied Mathematics (SIAM), Philadelphia, PA, 2002. https://doi.org/10.1137/1.9780898719208. Reprint of the 1978 original [North-Holland, Amsterdam; MR0520174 (58 \#25001)]

[9] A. Ern and J-L. Guermond. Theory and practice of finite elements, volume 159 of Applied Mathematical Sciences. Springer-Verlag, New York, 2004. https://doi.org/10.1007/978-1-4757-4355-5.

[10] Jr. Kuttler and L. Kenneth. A degenerate nonlinear Cauchy problem. Applicable Anal., 13(4):307-322, 1982. https://doi.org/10.1080/00036818208839402. 
[11] Jr. Kuttler and L. Kenneth. The Galerkin method and degenerate evolution equations. J. Math. Anal. Appl., 107(2):396-413, 1985. https://doi.org/10.1016/0022-247X(85)90321-X.

[12] Jr. Kuttler and L. Kenneth. Time-dependent implicit evolution equations. Nonlinear Anal., 10(5):447-463, 1986. https://doi.org/10.1016/0362546X(86)90050-7.

[13] R.C. MacCamy and M. Suri. A time-dependent interface problem for two-dimensional eddy currents. Quart. Appl. Math., 44(4):675-690, 1987. https://doi.org/10.1090/qam/872820.

[14] F. Paronetto. Existence results for a class of evolution equations of mixed type. $J$. Funct. Anal., 212(2):324-356, 2004. https://doi.org/10.1016/j.jfa.2004.03.014.

[15] F. Paronetto. Homogenization of degenerate elliptic-parabolic equations. Asymptot. Anal., 37(1):21-56, 2004.

[16] V. Pluschke. Solution of a quasilinear parabolic-elliptic boundary value problem. In Evolution equations and their applications in physical and life sciences (Bad Herrenalb, 1998), volume 215 of Lecture Notes in Pure and Appl. Math., pp. 265-276. Dekker, New York, 2001. https://doi.org/10.1201/9780429187810-22.

[17] A. Quarteroni and A. Valli. Numerical approximation of partial differential equations, volume 23 of Springer Series in Computational Mathematics. SpringerVerlag, Berlin, 1994. https://doi.org/10.1007/978-3-540-85268-1.

[18] W. Rudin. Functional analysis. International Series in Pure and Applied Mathematics. McGraw-Hill, Inc., New York, second edition, 1991.

[19] R.E. Showalter. Degenerate evolution equations and applications. Indiana Univ. Math. J., 23:655-677, 1975. https://doi.org/10.1512/iumj.1974.23.23056.

[20] R.E. Showalter. Monotone operators in Banach space and nonlinear partial differential equations, volume 49 of Mathematical Surveys and Monographs. American Mathematical Society, Providence, RI, 1997.

[21] V. Thomée. Galerkin finite element methods for parabolic problems, volume 25 of Springer Series in Computational Mathematics. Springer-Verlag, Berlin, second edition, 2006.

[22] A. Ženíšek. Nonlinear elliptic and evolution problems and their finite element approximations. Computational Mathematics and Applications. Academic Press, Inc. [Harcourt Brace Jovanovich, Publishers], London, 1990. With a foreword by P.-A. Raviart

[23] E. Zeidler. Nonlinear functional analysis and its applications. II/A. SpringerVerlag, New York, 1990. Linear monotone operators, Translated from the German by the author and Leo F. Boron.

[24] M. Zlámal. Finite element solution of quasistationary nonlinear magnetic field. RAIRO Anal. Numér., 16(2):161-191, 1982. https://doi.org/10.1051/m2an/1982160201611.

[25] M. Zlámal. Addendum to the paper: "Finite element solution of quasistationary nonlinear magnetic field" [RAIRO Anal. Numér. 16 (1982), no. 2, 161191; MR0661454 (83k:65086)]. RAIRO Anal. Numér., 17(4):407-415, 1983. https://doi.org/10.1051/m2an/1983170404071. 